\title{
Potential Mechanisms of Action of Chinese Patent Medicines for COVID-19: A Review
}

OPEN ACCESS

Edited by:

Patrícia Mendonça Rijo,

Universidade Lusófona, Portugal

Reviewed by:

Shih-Jie Chou,

Taipei Veterans General Hospital,

Taiwan

Guang-Bo Ge,

Shanghai University of Traditional

Chinese Medicine, China

Dayi Zhang,

Tsinghua University, China

*Correspondence:

Li-Min Feng

flimintcm@163.com

Hai-Dong Sun

shd02@163.com.com

${ }^{+}$These authors have contributed equally to this work and share first

authorship.

Specialty section:

This article was submitted to

Ethnopharmacology,

a section of the journa

Frontiers in Pharmacology

Received: 16 February 2021 Accepted: 08 June 2021

Published: 15 July 2021

Citation:

Yang $Z-H$, Wang $B, M a Q$, Wang $L$, Lin $Y-X$, Yan H-F, Fan Z-X, Chen H-J, Ge Z, Zhu F, Wang H-J, Zhang B-N,

Sun $H-D$ and Feng L-M (2021)

Potential Mechanisms of Action of Chinese Patent Medicines for COVID-

19: A Review.

Front. Pharmacol. 12:668407.

doi: 10.3389/fphar.2021.668407
Zhi-Hua Yang ${ }^{1,2 \dagger}$, Bin Wang ${ }^{2 \dagger}$, Qian Ma ${ }^{1,2}$, Lin Wang ${ }^{1,2}$, Ya-Xin Lin ${ }^{2}$, Hai-Feng Yan ${ }^{1,2}$, Zi-Xuan Fan ${ }^{1,2}$, Hao-Jia Chen ${ }^{1,2}$, Zhao Ge ${ }^{1,2}$, Feng Zhu ${ }^{3}$, Hui-Jie Wang ${ }^{4}$, Bao-Nan Zhang ${ }^{4}$, Hai-Dong Sun ${ }^{5,6 *}$ and Li-Min Feng ${ }^{7 *}$

${ }^{1}$ First Teaching Hospital of Tianjin University of Traditional Chinese Medicine, Tianjin, China, ${ }^{2}$ Tianjin University of Traditional Chinese Medicine, Tianjin, China, ${ }^{3}$ Department of Traditional Chinese Medicine, Hebei North University, Zhangjiakou, China, ${ }^{4}$ Department of Cardiology, Traditional Chinese Medicine Hospital of Tianjin Beichen District, Tianjin, China, ${ }^{5}$ Tianjin Fourth Central Hospital, Tianjin, China, ${ }^{6}$ Shenzhen Hospital Futian of Guangzhou University of Chinese Medicine, Shenzhen, China, ${ }^{7}$ Second Affiliated Hospital of Tianjin University of Traditional Chinese Medicine, Tianjin, China

Coronavirus disease 2019 (COVID-19) is an emergent infectious pneumonia caused by severe acute respiratory syndrome coronavirus 2 (SARS-CoV-2), which is highly contagious and pathogenic. COVID-19 has rapidly swept across the world since it was first discovered in December 2019 and has drawn significant attention worldwide. During the early stages of the outbreak in China, traditional Chinese medicines (TCMs) were involved in the whole treatment process. As an indispensable part of TCM, Chinese patent medicines (CPMs) played an irreplaceable role in the prevention and treatment of this epidemic. Their use has achieved remarkable therapeutic efficacy during the period of medical observation and clinical treatment of mild, moderate, severe, and critical cases and during convalescence. In order to better propagate and make full use of the benefits of TCM in the treatment of COVID-19, this review will summarize the potential target of SARS-CoV-2 as well as the theoretical basis and clinical efficacy of recommended 22 CPMs by the National Health Commission and the Administration of TCM and local provinces or cities in the treatment of COVID-19. Additionally, the study will further analyze the drug composition, potential active ingredients, potential targets, regulated signaling pathways, and possible mechanisms for COVID-19 through anti-inflammatory and immunoregulation, antiviral, improve lung injury, antipyretic and organ protection to provide meaningful information about the clinical application of CPMs.

Keywords: COVID-19, SARS-CoV-2, traditional Chinese medicine, Chinese patent medicine, review

Abbreviations: COVID-19, coronavirus disease 2019; SARS-CoV-2, severe acute respiratory syndrome coronavirus 2; TCM, traditional Chinese medicine; CPMs, Chinese patent medicines; WHO, World Health Organization; ICTV, International Committee on Taxonomy of Viruses; SARS, severe acute respiratory syndrome; MERS, Middle East respiratory syndrome; PHEIC, Public Health Emergency of International Concern; 3CLpro, 3-chymotrypsin-like protease; ACE2, angiotensinconverting enzyme 2; NSP, nonstructural proteins; ARDS, acute respiratory distress syndrome; AT2, alveolar type 2; GM-CSF, granulocyte-macrophage colony-stimulating factor; COX, cyclooxygenase; AA, arachidonic acid; IL, interleukin; IFN, interferon; C-X-C, C-X-C motif chemokine; Brd2, bromine domain containing protein 2; APN, aminopeptidase N. 


\section{INTRODUCTION}

In December 2019, the first cases of unexplained viral pneumonia were reported in Wuhan, Hubei Province, China. According to the results of etiologic examinations, the pathogen that caused the disease was a novel coronavirus (Chan et al., 2020). On February 11,2020 , the novel coronavirus disease was officially named coronavirus disease 2019 (COVID-19) by the WHO (World Health Organization, 2020). On the same day, the International Committee on Taxonomy of Viruses (ICTV) gave the novel coronavirus its current name severe acute respiratory syndrome coronavirus 2 (SAR-CoV-2) (Coronaviridae Study Group of the International Committee on Taxonomy of Viruses, 2020). COVID-19 is an acute infectious disease caused by SARS-CoV-2 and is subsequent to severe acute respiratory syndrome (SARS) and Middle East respiratory syndrome (MERS) (Paules et al., 2020). It is highly infectious and is disseminating quickly worldwide. Lack of sufficient knowledge led to the rapid spread of the epidemic in the early stages. On January 30, 2020, the WHO declared the COVID-19 epidemic as a Public Health Emergency of International Concern (PHEIC) (World Health Organisation, 2020). In the subsequent months, the ongoing outbreak of COVID-19 progressed quickly and globally. The WHO defined the severity of the COVID-19 outbreak to be a global "pandemic" on March 12, 2020 (World Health Organisation, 2020). So far, COVID-19 has been mainly clinically treated by symptomatic therapies, supportive therapies, and symptom improvement, and at present, a few vaccines have been developed (Centers for Disease Control and Prevention, 2020). As one of the earliest countries to contain and tackle COVID-19, China has taken prompt and effective measures including isolation, elimination, personal protection, and treatment approaches using traditional Chinese medicine (TCM), Western medicine therapy, and a combination of the latter. In particular, in the absence of specific drugs and vaccines (Guo et al., 2020b), TCM has played an important role in the prevention and treatment of COVID-19 at various stages due to its characteristics of syndrome differentiation and treatment, integrated participation, and prevention before the disease escalates. In China, $92.58 \%$ of cases were treated by TCM, and the number of cases in which TCM participated in the clinical therapy and discharge has exceeded $70 \%$ with the proportion continuously increasing (State Administration of Traditional Chinese Medicine, 2020). The outbreak of COVID-19 has been well controlled in China for now, and TCM has contributed greatly (Xu and Zhang, 2020b). In particular, Chinese patent medicines (CPMs) have played an irreplaceable role and have provided unique advantages in the management of COVID-19. However, the underlying mechanisms of CPMs are still unclear. This review will summarize and analyze the possible active mechanisms of CPMs in the treatment of COVID-19. The findings reported may provide meaningful information for further study to investigate the mechanisms of CPMs as a therapeutic approach to overcoming COVID-19. Figure 1 shows the framework of this review.

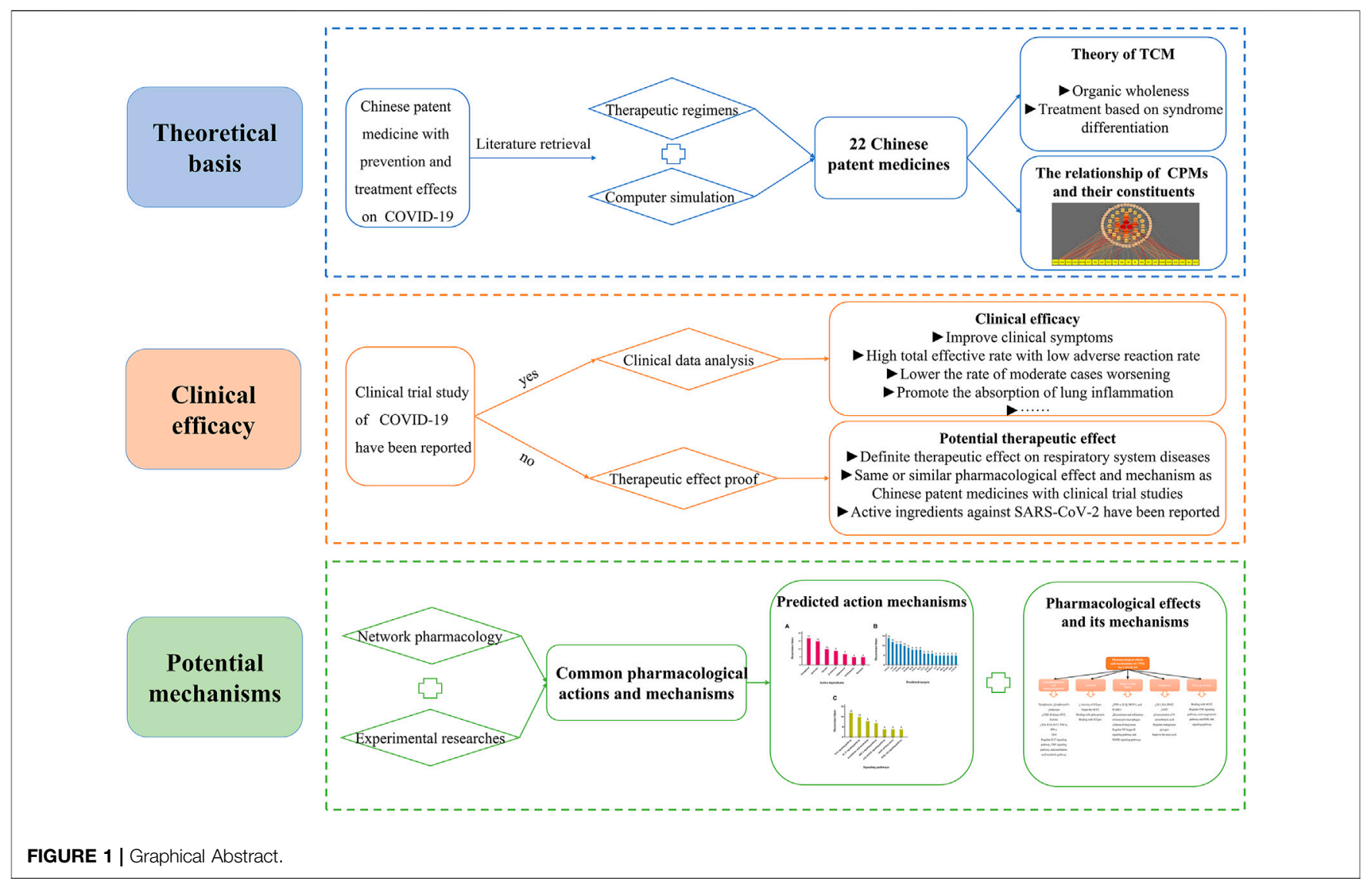




\section{POTENTIAL TARGET FOR THE PREVENTION AND INHIBITION OF SEVERE ACUTE RESPIRATORY SYNDROME CORONAVIRUS 2}

SARS-CoV-2 is composed of four structural proteins and nonstructural proteins (NSP) (Gallagher and Buchmeier, 2001). Its structural proteins play an important role in the assembly of viruses and infection of hosts. Among them, the Spike (S) trimer is highly glycosylated to form the spikes on the surface of the virus, which is responsible for binding to the host cell receptor and allows the coronavirus to invade the host cell (Snijder et al., 2003). Studies have shown that angiotensin-converting enzyme 2 (ACE2) is a cell receptor which SARS-CoV-2 S glycoprotein can bind to (Zhou et al., 2020a). Alveolar type 2 (AT2) cells are the primary host cell of viral invasion due to it expressing higher levels of ACE2 protein. AT2 cells express higher levels of ACE2 protein. Viral replication induces an immune response in the body and produces excessive cytokines resulting in the formation of a cytokine storm after SARS-CoV-2 has entered the alveolar epithelial cells. This would cause inflammation and in turn lead to lung injury, acute respiratory distress syndrome (ARDS), and multiple organ failure and even death (Huang et al., 2020). Therefore, ACE2 is also a target molecule for lung damage caused by the virus (Kuba et al., 2005). Furthermore, cells in the heart, kidney, and other organs may become targets of viral invasion because of their high expression of ACE2. This will lead to damage to the lung, heart, kidney, and other important organs (Leng et al., 2020). Thus, disrupting the interaction between the SARS-CoV-2 S glycoprotein and this enzyme would prevent viral invasion. Nonstructural proteins of coronavirus participate in the transcription and replication of viral genomes, most of which are 3-chymotrypsin-like protease (3CLpro), Papain-like protease (PLpro), helicase, and 3-5' exonuclease (Smith et al., 2013). The responsibility of SARS-CoV-2 3CLpro is to cleave polyproteins and generate functional enzymes. Without it, the entire replication architecture will not work properly (Loschwitz et al., 2021). So 3CLpro is considered to be a necessary target in the process of viral replication. Collectively, ACE2 and SARS-CoV-2 3CLpro are important targets for the prevention and inhibition of the virus, whose activity involves the invasion of host cells and intracellular replication of the virus (Song et al., 2018; Li et al., 2020a; Chen Y. W. et al., 2020; Wrapp et al., 2020).

\section{THEORETICAL BASIS AND CLINICAL EFFICACY OF TRADITIONAL CHINESE MEDICINE IN THE PREVENTION AND TREATMENT OF CORONAVIRUS DISEASE 2019}

In the theory of TCM, COVID-19 belongs to the category of "plague," given its feature of strong infectiousness and the resulting rapid epidemic. Exogenous pathogenic epidemic is the main etiology, and the virus target location is the lung. In addition, the basic pathogenesis is characterized by "dampness, heat, toxin, and stasis" (Chen et al., 2020a). Since ancient times, experts of TCM have accumulated rich clinical experience in the treatment of plagues. The advantage of TCM is that even if the pathogen is not clear, a set of corresponding prescriptions can be proposed based on clinical syndromes under the guidance of the theory of syndrome differentiation and treatment, which can alleviate the disease, shorten its course, and attenuate worsening or complications (Yang, 2020b). In this epidemic, TCM has exerted beneficial effects in a comprehensive way during the treatment of COVID-19. The concept of organic wholeness and the characteristics of treatment based on syndrome differentiation have contributed greatly to the therapy of COVID-19, especially in improving patients' clinical symptoms, which is of great significance in enhancing survival rates (Li et al., 2020c).

At present, "Diagnosis and Treatment Protocol for COVID-19 (trial version 8)" formulated by the National Health Commission and the Administration of TCM and Chinese medicine prevention recommend 13 Chinese patent medicines for different stages (National Health Commission of the People's Republic of China and National Administration of Traditional Chinese Medicine, 2020). During the medical observation period, the clinical manifestations were fatigue with gastrointestinal discomfort, and Huoxiang Zhengqi Capsule (pill, liquid, and oral liquid) was recommended. For the clinical manifestation of fatigue with fever, the recommended Chinese patent medicines are Jinhua Qinggan Granule, Lianhua Qingwen Capsule (granule), and Shufeng Jiedu Capsule (granule). During the clinical treatment period, Qingfei Paidu decoction can be used in the treatment of mild, moderate, and severe cases and can be used reasonably with the consideration of the actual conditions of critically ill patients. In addition, the corresponding prescriptions were recommended for different syndromes of mild, moderate, severe, and critical patients; Xiyanping Injection, Xuebijing Injection, Reduning Injection, Shengmai Injection, Shenfu Injection, Shenmai Injection, Tanreqing Injection, and Xingnaojing Injection are recommended for severe and critical patients. Besides, seven provincial and municipal diagnosis and treatment protocols involving nine types of CPMs were retrieved. The above 22 types of CPMs have been recommended as treatment schemes for the potential treatment of COVID-19 by using network pharmacology and molecular docking technology. The information of $22 \mathrm{CPMs}$ is provided in Table 1. The details involving main findings, study design, severity classification, course of treatment, and clinical data for recommended CPMs are summarized in Table 2. Further, the relationship of CPMs and their constituents is depicted in Figure 2.

Table 2 shows ten CPMs recommended for the treatment of COVID-19, including 14 completed studies in our review. Among 14 studies, three studies involved mild patients, seven studies involved ordinary patients, two studies involved both mild and ordinary patients, only one study involved severe patients, and one study did not report the stage of severity of COVID- 19 . There are 15 indexes used to evaluate the therapeutic effect of Chinese patent medicines. The total effective rate, aggravation 
TABLE 1 | Therapeutic regimens of recommended CPMs for COVID-19 in China.

\begin{tabular}{lll}
\hline No & $\begin{array}{l}\text { Name of Chinese } \\
\text { patent medicines }\end{array}$ & \multicolumn{1}{c}{ Clinical stage } \\
\hline $1 \quad \begin{array}{l}\text { Lianhua Qingwen } \\
\text { Capsule }\end{array}$ & Medical observation period \\
$2 \quad$ & $\begin{array}{l}\text { Jinhua Qinggan } \\
\text { Granules }\end{array}$ & Medical observation period \\
& $\begin{array}{l}\text { Shufeng Jiedu } \\
\text { Capsule }\end{array}$ & Medical observation period \\
&
\end{tabular}

$4 \quad$ Huoxiang Zhengqi

Medical observation period Oral Capsule

5 Qingfei Paidu decoction

6 Xiyanping Injection

$7 \quad$ Xuebijing Injection

$8 \quad$ Xingnaojing Injection

$9 \quad$ Reduning Injection

10 Tanreqing Injection

11 Shenmai Injection

12 Shengmai Injection

13 Shenfu Injection

14 Kangbingdu Granules

15 Shuanghuanglian Oral Liquid

16 Reyanning Mixture

17 Siji Kangbingdu Mixture
Clinical treatment period-mild, moderate, severe, and critical cases

Clinical treatment period-severe case

Clinical treatment period - severe and critical cases

Clinical treatment

period-severe and critical cases

Clinical treatment period - severe and critical cases

Clinical treatment period-severe and critical cases

Clinical treatment period-critical case

Clinical treatment period - critical case

Clinical treatment period-critical case

Medical observation period; clinical treatment period-mild case

Medical observation period; clinical treatment period-mild and moderate cases

Clinical treatment period-mild case

Clinical treatment period-mild case

\section{Therapeutic regimen of} COVID-19

National Health Commission of the People's Republic of China. Guideline on Diagnosis and Treatment of COVID-19 (trial 8th edition)

National Health Commission of the People's Republic of China. Guideline on Diagnosis and Treatment of COVID-19 (trial 8th edition)

National Health Commission of the

People's Republic of China. Guideline on Diagnosis and Treatment of COVID-19 (trial 8th edition)

National Health Commission of the

People's Republic of China. Guideline on Diagnosis and Treatment of COVID-19 (trial 8th edition)

National Health Commission of the People's Republic of China. Guideline on Diagnosis and Treatment of COVID-19 (trial 8th edition)

National Health Commission of the

People's Republic of China. Guideline on Diagnosis and Treatment of COVID-19 (trial 8th edition)

National Health Commission of the

People's Republic of China. Guideline on Diagnosis and Treatment of COVID-19 (trial 8th edition)

National Health Commission of the

People's Republic of China. Guideline on Diagnosis and Treatment of COVID-19 (trial 8th edition)

National Health Commission of the People's Republic of China. Guideline on Diagnosis and Treatment of COVID-19 (trial 8th edition)

National Health Commission of the People's Republic of China. Guideline on Diagnosis and Treatment of COVID-19 (trial 8th edition)

National Health Commission of the People's Republic of China. Guideline on Diagnosis and Treatment of COVID-19 (trial 8th edition)

National Health Commission of the

People's Republic of China. Guideline on Diagnosis and Treatment of COVID-19 (trial 8th edition)

\section{National Health Commission of the}

People's Republic of China. Guideline on Diagnosis and Treatment of COVID-19 (trial 8th edition)

Guangdong Provincial COVID-19 TCM

Therapeutic Regime (trial version II)

Beijing Municipal COVID-19 TCM

Preventive and Therapeutic Regime (trial version V)

Shanxi provincial COVID-19 TCM

therapeutic regime (trial version II)

Shanxi Provincial COVID-19 TCM

Therapeutic Regime (trial version II) http://www.nhc.gov.cn/cms-search/xxgk/

getManuscriptXxgk.htm?

id=0a7bdf12bd4b46e5bd28ca7f9a7f5e5a

http://www.nhc.gov.cn/cms-search/xxgk/ getManuscriptXxgk.htm?

id=0a7bdf12bd4b46e5bd28ca7f9a7f5e5a

http://www.nhc.gov.cn/cms-search/xxgk/ getManuscriptXxgk.htm?

id=0a7bdf12bd4b46e5bd28ca7f9a7f5e5a

http://www.nhc.gov.cn/cms-search/xxgk/ getManuscriptXxgk.htm?

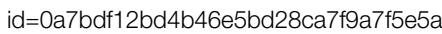

http://www.nhc.gov.cn/cms-search/xxgk/ getManuscriptXxgk.htm?

id=0a7bdf12bd4b46e5bd28ca7f9a7f5e5a

http://www.nhc.gov.cn/cms-search/xxgk/ getManuscriptXxgk.htm?

id=0a7bdf12bd4b46e5bd28ca7f9a7f5e5a

http://www.nhc.gov.cn/cms-search/xxgk/ getManuscriptXxgk.htm?

id=0a7bdf12bd4b46e5bd28ca7f9a7f5e5a

http://www.nhc.gov.cn/cms-search/xxgk/ getManuscriptXxgk.htm?

id=0a7bdf12bd4b46e5bd28ca7f9a7f5e5a

http://www.nhc.gov.cn/cms-search/xxgk/ getManuscriptXxgk.htm?

id $=0 a 7 b d f 12 b d 4 b 46 e 5 b d 28 c a 7 f 9 a 7 f 5 e 5 a$

http://www.nhc.gov.cn/cms-search/xxgk/ getManuscriptXxgk.htm?

id=0a7bdf12bd4b46e5bd28ca7f9a7f5e5a

http://www.nhc.gov.cn/cms-search/xxgk/ getManuscriptXxgk.htm?

id=0a7bdf12bd4b46e5bd28ca7f9a7f5e5a

http://www.nhc.gov.cn/cms-search/xxgk/ getManuscriptXxgk.htm?

id=0a7bdf12bd4b46e5bd28ca7f9a7f5e5a

http://www.nhc.gov.cn/cms-search/xxgk/ getManuscriptXxgk.htm?

id=0a7bdf12bd4b46e5bd28ca7f9a7f5e5a

http://szyyj.gd.gov.cn/_wgk/gsgg/content/post_2902010. $\mathrm{html}$

http://zyj.beijing.gov.cn/sy/tzgg/202006/t20200616_ 1926196.html

http://atcm.shaanxi.gov.cn/sy/tzgg/202002/t20200202_ 2048353.html

http://atcm.shaanxi.gov.cn/sy/tzgg/202002/t20200202_ 2048353.html

(Continued on following page) 
TABLE 1 | (Continued) Therapeutic regimens of recommended CPMs for COVID-19 in China.

\begin{tabular}{|c|c|c|c|c|}
\hline No & $\begin{array}{l}\text { Name of Chinese } \\
\text { patent medicines }\end{array}$ & Clinical stage & $\begin{array}{l}\text { Therapeutic regimen of } \\
\text { COVID-19 }\end{array}$ & Website \\
\hline 18 & $\begin{array}{l}\text { Qingxuan Zhike } \\
\text { Granules }\end{array}$ & $\begin{array}{l}\text { Clinical treatment period-mild } \\
\text { case }\end{array}$ & $\begin{array}{l}\text { Hunan Provincial COVID-19 newborn } \\
\text { infants Preventive and Therapeutic } \\
\text { Regime (trial version I) }\end{array}$ & http://www.hnysfww.com/article.php?id = 1820 \\
\hline 19 & Jinzhen Oral Liquid & $\begin{array}{l}\text { Clinical treatment period-mild } \\
\text { and moderate cases }\end{array}$ & $\begin{array}{l}\text { The 24th press conference on COVID-19 } \\
\text { prevention and control held by the } \\
\text { Information Office of Guangdong } \\
\text { Provincial Government }\end{array}$ & $\begin{array}{l}\text { http://www.gd.gov.cn/gdywdt/zwzt/yqfk/fbh/content/post_ } \\
\text { 2924024.html }\end{array}$ \\
\hline 20 & Jinye Baidu Granules & $\begin{array}{l}\text { Clinical treatment period-mild } \\
\text { and moderate cases }\end{array}$ & $\begin{array}{l}\text { TCM Diagnosis and Treatment Scheme } \\
\text { and Preventive Scheme for Novel } \\
\text { Coronavirus Infected Pneumonia of Tongji } \\
\text { Hospital, Tongji Medical College, } \\
\text { Huazhong University of Science \& } \\
\text { Technology }\end{array}$ & https://www.tjh.com.cn/html/2020/0208/28991.shtml \\
\hline 21 & Qingkailing Injection & $\begin{array}{l}\text { Clinical treatment } \\
\text { period-severe case }\end{array}$ & $\begin{array}{l}\text { TCM Diagnosis and Treatment Scheme } \\
\text { and Preventive Scheme for Novel } \\
\text { Coronavirus Infected Pneumonia of Tongji } \\
\text { Hospital, Tongji Medical College, } \\
\text { Huazhong University Of Science \& } \\
\text { Technology }\end{array}$ & https://www.tjh.com.cn/html/2020/0208/28991.shtml \\
\hline 22 & $\begin{array}{l}\text { Bufei Huoxue } \\
\text { Capsule }\end{array}$ & Convalescence & $\begin{array}{l}\text { Guizhou Provincial COVID-19 TCM } \\
\text { Preventive and Therapeutic Reference } \\
\text { Regime (version II) }\end{array}$ & $\begin{array}{l}\text { http://atcm.guizhou.gov.cn/xwzx/tzgg/202002/ } \\
\text { t20200219_50073831.html }\end{array}$ \\
\hline
\end{tabular}

rate, and adverse reaction rate are mainly used to evaluate the efficacy and safety of Chinese patent medicines. In addition, it also involves the disappearance rate of main symptoms, the duration of main symptoms, improvement rate of pulmonary $\mathrm{CT}$, recovery rate of inflammation level, negative conversion rate (time) of nucleic acid, and other secondary indexes. The result shows the total effective rates of LHQW (Cheng et al., 2020), HXZQ (Yang et al., 2020a), QFPD (Wang et al., 2021), and TRQ (Huang et al., 2020b) were above $85 \%$ and the treatment effect is obvious. Although the total effective rate of Xuebijing was only $68.2 \%$, there was a significant difference compared with the control group. The adverse reaction rate and the aggravation rate were low, less than 10\% (Cheng et al., 2020; Yang H. B. et al., 2020; Yu H. Y. et al., 2020; Lu et al., 2020; Wang et al., 2021), indicating that those CPMs can improve the efficacy with a good safety profile. These clinical trials found that these drugs can significantly improve the clinical symptoms caused by COVID19. For patients of mild and moderate cases, it is necessarily important to improve the main symptoms such as fever, cough, fatigue, and expectoration, which avoid the transformation from mild to severe. CPMs contribute the most to the improvement of fever symptoms, the disappearance rate of fever is more than $80 \%$ (Duan et al., 2020), and the duration of fever was shorter in the treatment group (Cheng et al., 2020; Lu et al., 2020; Yang, 2020b). However, CPMs in relieving cough symptoms are weak, and the disappearance rate of cough is only 55\% (Lu et al., 2020). By observing the improvement rate of pulmonary CT and the recovery level of inflammatory indexes, we can further know the therapeutic effect of CPMs on COVID-19. SFJD (Chen et al., 2020c), QFPD (Zeng et al., 2020), and XBJ (Zhang et al., 2020a) can significantly improve the absorption of lung CT lesions, LHQW (Cheng et al., 2020) and RYN (Yang et al., 2020c) also have this therapeutic effect, but there is no significant difference. Before and after the treatment, the difference of the oxygenation indexes and inflammatory factors such as white blood cell (WBC), lymphocytes (LYMPH), erythrocyte sedimentation rate (ESR), interleukin (IL), tumor necrosis factor- $\alpha$ (TNF- $\alpha$ ), C-reactive protein (CRP), and procalcitonin (PCT) was compared between the two groups, which in the treatment group was better than that in the control group (Liu K. F. et al., 2020; Qin et al., 2020; Chen et al., 2020c; Wang et al., 2021). Besides, SFJD (Qu et al., 2020), QFPD (Zeng et al., 2020), and RYN (Yang et al., 2020c) can shorten the negative conversion rate (time) of nucleic acid.

At present, the randomized controlled trials of some drugs have not been completed (such as Shengmai Injection and Bufei Huoxue Capsule) or are still in progress (such as Xiyanping Injection and Shuanghuanglian Oral Liquid), so the effectiveness of drugs in the treatment of COVID-19 cannot be determined temporarily (Wang et al., 2020a). However, because of the need for treatment, the above drugs have been widely used in the treatment of patients with COVID-19 in accordance with the strategy of "New uses of old drugs" and "Treating different diseases with the same method" in the TCM theory. The active component of the Xiyanping Injection is the total sulfonate of andrographolide, and it has the function of clearing heat and detoxification and antibacterial and antiinflammatory and definite therapeutic effect on respiratory system diseases (Cai et al., 2020). Shuanghuanglian Oral Liquid (Zheng et al., 2020b) and Xingnaojing Injection (Xie et al., 2020) also have the function of regulating inflammatory factors and relieving inflammation reaction. This is one of the therapeutic mechanisms of COVID-19, which was treated by avoiding the cytokine storm. Shenfu Injection, Shengmai Injection, and Shenmai Injection can enhance the immune function and 
TABLE 2 | Summary of clinical efficacy of CPMs for COVID-19.

\begin{tabular}{ll} 
No $\quad \begin{array}{c}\text { Name of } \\
\text { Chinese } \\
\text { patent } \\
\text { medicines }\end{array}$ & \multicolumn{1}{c}{ Main findings } \\
\hline $1 \quad$ Lianhua Qingwen & $\begin{array}{l}\text { (1) Improve clinical symptoms of fever, fatigue, } \\
\text { cough, expectoration, shortness of breath, } \\
\text { moist crackles, chest distress, and appetite } \\
\text { loss. (2) Enhance the effective rate of cardinal } \\
\text { symptom. (3) Reduce the proportion of normal } \\
\text { to heavy. }\end{array}$
\end{tabular}

symptom. (3) Reduce the proportion of normal

2 Jinhua Qinggan Granules (JHQG) fever, cough, fatigue, and expectoration. (2) Relieve the anxiety of patients. the absorption of lung inflammation. (4) Significantly shorten the symptoms improvement time and negative conversion time of the clinical.

$4 \quad$ Huoxiang Zhengqi Oral Capsule (HXZQ)

(1) Significantly improve clinical symptoms such as fever, cough, fatigue, and white greasy coating. (2) Lower the rate of moderate cases worsening. (3) Improve the clinical cure rate.

5 Qingfei Paidu decoction (QFPD)
(1) Significantly reduce the clinical symptoms of
(1) Significantly improve clinical symptoms and TCM syndrome. (3) Improve the negative psychology and decrease risks of complications.

\author{
Retrospective study; LHQW group: LHQW + \\ conventional western therapy $(n=63)$ vs. contro \\ group: conventional western therapy $(n=38)$ \\ Retrospective study; LHQW group: LHQW + \\ conventional western therapy ( $n=51$ ) vs. contro
}

Medica

observation

period group: conventional western therapy $(n=51)$
Randomized controlled trial; JHQG group: JHQG + conventional western therapy $(n=82)$ vs. control group: conventional western therapy $(n=41)$

Retrospective study; SFJD group: SFJD + arbidol hydrochloride capsule $(n=40)$ vs. control group: arbidol hydrochloride capsule $(n=30)$

Retrospective study; SFJD group: SFJD + conventional western therapy $(n=34)$ vs. control group: conventional western therapy $(n=34)$

Case series; COVID-19 patients treated by HXZQ and conventional western therapy $(n=11)$

Randomized controlled trial QFPD group: QFPD + conventional western therapy $(n=70)$ vs. control group: conventional western therapy $(n=70)$

Retrospective study; QFPD group: QFPD conventional western therapy $(n=104)$ vs. contro group: conventional western therapy $(n=125)$
Mild

5 days

Mild and ordinary $\quad 10$ days Ordinary

7 days

(2)
(5) Shortness of breath: LHQW $68.2 \%$ vs. control $20.0 \%, p<0.05$; moist crackles: LHQW $56.0 \%$ vs. control $20.0 \%, p<0.05$

(10) Fever: LHQW 6 days vs. control 7 days, $p=0.17$

(44) LHQW $6.40 \%$ vs. control $15.8 \%, p>0.05$ (1) LHQW $83.7 \%$ vs. control $61.0 \%, p<0.05$ (2) LHQW $61.3 \%$ vs. control $34.3 \%, p<0.05$ (3) LHQW $62.2 \%$ vs. control $35.9 \%, p<0.05$ (5) Expectoration: LHQW $55.0 \%$ vs. control $15.8 \%$; shortness of breath: LHQW $61.5 \%$ vs. control $14.3 \%, p<0.05$; chest distress: LHQW $54.6 \%$ vs. control $15.8 \%, p<0.05$; appetite loss: LHQW $34.8 \%$ vs. control $7.70 \%, p<0.05$ (6) LHQW $54.9 \%$ vs. control $45.1 \%, p>0.05$ (12) LHQW $86.3 \%$ vs. control $68.6 \%, p<0.05$ (44) LHQW $7.80 \%$ vs. control $21.6 \%, p<0.05$ (1) JHQG $80.3 \%$ vs. control $53.1 \%, p=0.0050$ (2) JHQG $66.1 \%$ vs. control $42.9 \%, p=0.038$ (3) JHQG $77.6 \%$ vs. control $53.8 \%, p=0.028$ (4) JHQG $85.3 \%$ vs. control $46.2 \%, p=0.017$ (11) Compared with the control group, JHQG group significantly decrease, $p<0.01$ (8) SFJD $11.9 \pm 3.21$ vs. control $9.32 \pm 3.03$, $p<0.05$

(1) Compared with the control group, dry cough, nasal congestion, runny nose, pharyngeal pain, fatigue and diarrhea significantly improved $(p<0.05)$ in the SFJD group

(2) SFJD $91.3 \%$ vs. control $54.2 \%, p<0.05$ (3) SFJD $100 \%$ vs. control $70.6 \%, p<0.05>$ (4) SFJD $100 \%$ vs. control $37.5 \%, p<0.05$ (5) Chest distress: SFJD $100 \%$ vs. control. $57.1 \%, p<0.05$; shortness of breath: SFJD $90.9 \%$ vs. control $45.5 \%, p<0.05$

(6) SFJD $91.2 \%$ vs. control $70.6 \%, p<0.05$ (7) The level of lymphocytes significantly increased $(p<0.05)$ and the level of $\mathrm{C}$-reactive protein significantly decreased $(p<0.05)$ in both two groups; while procalcitonin and D-dimer significantly decreased $(p<0.05)$ in the SFJD group

(1) $100 \%$

(2) $60.0 \%$

(3) $100 \%$

(5) $64.0 \%$

(2) $100 \%$

(4) $9.09 \%$

Ordinary 10 days

(7) QFPD $95.7 \%$ vs. control $85.7 \%, p<0.05$ (9) Compared with the control group, QFPD group significantly shorter, $p<0.05$

(12) QFPD $98.6 \%$ vs. control $90.0 \%, p<0.05$ (3) QFPD $1.43 \%$ vs. Control $12.9 \%, p<0.05$ (6) QFPD $89.4 \%$ vs. control $71.2 \%, p<0.001$ (8) QFPD $5.36 \pm 1.25$ vs. control $10.6 \pm 2.62$, $p<0.001$

(9) QFPD $24.6 \pm 2.31$ vs. control $29.4 \pm 2.47$, $p<0.001$

(11) Compared with the control group, QFPD group significantly decrease, $p<0.05$ (5) QFPD $51.0 \%$ vs. Control $70.4 \%, p=0.0040$

6 Xuebijing (1) Promote the absorption of pulmonary Injection (XBJ) infection.

(1) Improve the clinical symptoms of COVID-19 patients, such as sore throat, cough, fatigue, headache, and chest tightness. (2) Promote the improvement of chest CT. (3) Shorten the duration of fever and improve the novel coronavirus nucleic acid conversion rate.
Retrospective study; XBJ group: XBJ + conventional western therapy $(n=22)$ vs. control group: conventional western therapy $(n=22)$ Randomized controlled trial; XBJ group: XBJ + conventional western therapy $(n=10)$ vs. control group: conventional western therapy $(n=10)$ Randomized controlled trial; RYN group: RYN + conventional western therapy $(n=26)$ vs. control group: conventional western therapy $(n=23)$

\begin{tabular}{|c|c|c|c|}
\hline & & & \\
\hline Ordinary & 7 days & $\begin{array}{l}\text { (6) XBJ } 95.5 \% \text { vs. control } 68.2 \%, p=0.017 \\
\text { (12) XBJ } 68.2 \% \text { vs. control } 50.0 \%, p=0.0010\end{array}$ & $\begin{array}{l}\text { Zhang et al. } \\
\text { (2020a) }\end{array}$ \\
\hline Mild & 3 days & $\begin{array}{l}\text { (7) The level of lymphocyte count, CRP, and } \\
\text { ESR in XBJ group were better than those of the } \\
\text { control group, } p>0.05\end{array}$ & $\begin{array}{l}\text { Liu et al. } \\
\text { (2020a) }\end{array}$ \\
\hline Ordinary & 7 days & $\begin{array}{l}\text { (1)-(5) Except cough and fatigue, other } \\
\text { symptoms disappeared in the RYN group, and } \\
\text { the disappearance rate of symptoms such as } \\
\text { dry throat, cough, fatigue, chest tightness, and } \\
\text { headache was statistically significant compared } \\
\text { with the control group }(p<0.05 \text { ) } \\
\text { (6) RYN } 88.5 \% \text { vs. control } 74.0 \%, p>0.05 \\
\text { (8) RYN } 96.2 \% \text { vs. control } 60.9 \%, p<0.01\end{array}$ & $\begin{array}{l}\text { Yang et al. } \\
\text { (2020c) }\end{array}$ \\
\hline
\end{tabular}

(Continued on following page) 
TABLE 2 | (Continued) Summary of clinical efficacy of CPMs for COVID-19.

\begin{tabular}{|c|c|c|c|c|c|c|c|}
\hline No & $\begin{array}{c}\text { Name of } \\
\text { Chinese } \\
\text { patent } \\
\text { medicines }\end{array}$ & Main findings & Study design & $\begin{array}{l}\text { Severity } \\
\text { classification }\end{array}$ & $\begin{array}{l}\text { Course of } \\
\text { treatment }\end{array}$ & Clinical data & References \\
\hline & & & & & & $\begin{array}{l}\text { (1) Fever: RYN } 5 \text { days vs. control } 3 \text { days, } \\
p>0.05\end{array}$ & \\
\hline 8 & $\begin{array}{l}\text { Jinye Baidu } \\
\text { Granules (JYBD) }\end{array}$ & $\begin{array}{l}\text { Lower the rate of moderate and mild cases } \\
\text { worsening }\end{array}$ & $\begin{array}{l}\text { Retrospective study; JYBD group: JYBD + } \\
\text { conventional western therapy }(n=27) \text { vs. Control } \\
\text { group: methylprednisolone + conventional } \\
\text { western therapy }(n=38)\end{array}$ & Mild and ordinary & $5-7$ days & (4) JYBD $7.40 \%$ vs. control $42.1 \%, p<0.05$ & $\begin{array}{l}\text { Yu et al. } \\
\text { (2020a) }\end{array}$ \\
\hline \multirow[t]{2}{*}{9} & $\begin{array}{l}\text { Reduning } \\
\text { Injection (RDN) }\end{array}$ & $\begin{array}{l}\text { (1) Significantly reduce the levels of } \\
\text { inflammatory factors }\end{array}$ & $\begin{array}{l}\text { Retrospective study; RDN group: RDN + } \\
\text { methylprednisolone }(n=21) \text { vs. control group: } \\
\text { methylprednisolone }(n=26)\end{array}$ & Severe & 5-7 days & $\begin{array}{l}\text { (7) Compared with control group after } \\
\text { treatment, the levels of WBC, IL6, IL17, and } \\
\text { CRP in RDN group were significantly lower }(p< \\
0.05) \text {, and the level of IL4 in group A was } \\
\text { significantly higher }(p<0.05)\end{array}$ & $\begin{array}{l}\text { Qin et al. } \\
(2020)\end{array}$ \\
\hline & & $\begin{array}{l}\text { (2) Reduce the length of hospital stay and ICU } \\
\text { stay }\end{array}$ & & & & $\begin{array}{l}\text { (9) The length of hospitalization in RDN group } \\
\text { was significantly shorter }(p<0.05)\end{array}$ & \\
\hline 10 & $\begin{array}{l}\text { Tanreqing } \\
\text { Injection (TRQ) }\end{array}$ & $\begin{array}{l}\text { Improve symptoms, block deterioration and } \\
\text { promote rehabilitation }\end{array}$ & $\begin{array}{l}\text { Retrospective study; TRQ group: TRQ }+\alpha- \\
\text { interferon + conventional western therapy }(n=58) \\
\text { vs. control group: conventional western therapy } \\
(n=60)\end{array}$ & $\begin{array}{l}\text { All confirmed } \\
\text { stages }\end{array}$ & 7 days & $\begin{array}{l}\text { (10) The symptom score in the TRQ group was } \\
\text { significantly higher }(p<0.05) \\
\text { (1) TRQ } 93.1 \% \text { vs. control } 80.0 \%, p<0.05\end{array}$ & $\begin{array}{l}\text { Huang et al. } \\
\text { (2020b) }\end{array}$ \\
\hline
\end{tabular}

Note: Clinical data (1)-(15): (1) disappearance rate of fever; (2) disappearance rate of cough; (3) disappearance rate of fatigue; (4) disappearance rate of expectoration; (5) disappearance rate of other signs and symptoms; (6) the improvement rate of pulmonary CT; (7) the recovery level of inflammatory indexes; (8) the negative conversion rate (time) of nucleic acid; (9) the length of hospitalization; (10) the duration of main symptoms; (11) the anxiety level; (12) total effective rate; (13) the adverse reaction rate; (14) aggravation rate; (15) complication rate.

hypoxia tolerance of myocardial cells, but the anti-inflammatory effect of Shengmai Injection is stronger than that of the other two (Zhang and Yang, 2008; Li et al., 2016). Early experiments in vitro antibiosis and in vivo animal studies showed (Wu et al., 2018) that Kangbingdu Granules had an antibacterial effect in vitro and antiviral, antipyretic, and anti-inflammatory effects in vivo. Liu et al. (2020b) conducted a meta-analysis for Siji Kangbingdu Mixture efficacy and safety in the treatment of children respiratory system infections. The result shows that Siji Kangbingdu Mixture can shorten the time of fever, cough, and sore throat, the efficacy effect and safety of Siji Kangbingdu Mixture were significantly better than those of ribavirin or interferon. Qingxuan Zhike Granules in the treatment of children with upper respiratory infection (Zhang et al., 2015) and cold of wind-heat (Du et al., 2021) can effectively improve the clinical symptoms, especially for cough, sputum, and pharyngeal symptoms. Jinzhen Oral Liquid (Yu, 2019) has antipyretic, anti-inflammatory, antitussive, and antiviral pharmacological effects, and it is mainly used in the treatment of children with various respiratory diseases in clinical. Qingkailing Injection can promote the release of antipyretic substances and regulate body temperature by inhibiting endogenous pyrogen and endotoxic fever (Zhao et al., 2019). Bufei Huoxue Capsules (Zhou et al., 2020b) can improve lung ventilation function, reduce blood viscosity, and increase blood oxygen content to play a role in the treatment of chronic respiratory diseases. These drugs can improve clinical efficiency and reduce the incidence of adverse reactions in respiratory diseases (State Administration of Traditional Chinese Medicine, 2020; Xu and Zhang, 2020b). But, due to differences in usage, dosage, and application population, the adverse reactions may have new changes. So clinical trials should be completed as soon as possible (Hu and Zhang, 2020).

Figure 2 shows that Lonicera japonica Thunb, Glycyrrhiza uralensis Fisch, Scutellaria baicalensis Georgi, Gardenia jasminoides Ellis, Isatidis Radix, Forsythia suspensa Vahl, and Agastache rugosa are frequently used among all the recommended CPMs (chose only if frequency $\geq 4$ for CPMs). These drugs are widely used in the treatment of COVID-19 for its function of clearing away heat and toxin (Chen et al., 2020b; Yu Y. R. et al., 2020). Zhou et al. (2021) suggested that Lonicera japonica Thunb extract significantly increased body weight, organ index, serum cytokines (interleukin, tumor necrosis factor, and interferon- $\gamma$ ), secretory immunoglobulin A ( $\operatorname{IgA})$, and the activity of natural killer (NK) cells and cytotoxic lymphocytes (CTL) of colitis ulcerative mouse. Besides, it can also reduce the apoptosis of splenic lymphocytes in colitis ulcerative mouse (Nomura et al., 2019). It has been found Glycyrrhizic acid can inhibit the replication of the virus and interfere with the cycle of virus adsorption and penetration. Yu et al. (2020b) indicated that Glycyrrhizic acid could inhibit the binding of RBD part of S protein and ACE2 by the MTT assay to play antiviral effect. Some researchers used Scutellaria baicalensis Georgi for antibacterial test in vitro. Some experiment results showed (Li et al., 2013) that Scutellaria baicalensis Georgi has a strong inhibitory effect on Staphylococcus aureus, Escherichia coli, Pseudomonas aeruginosa, and other viruses. Moreover, baicalein, an active ingredient of Scutellaria baicalensis Georgi, has a strong inhibitory effect on HIV. Gardenia jasminoides Ellis is an important medicine to cure epidemic diseases. It can significantly inhibit the lung inflammation of mice induced by influenza virus, significantly reduce the mortality of mice infected by influenza virus, prolong the survival time, and reduce the level of NO in serum. It also can inhibit the cytopathy caused by H1N1, PIV-1, RSV, HSV-1, and HSV-2 in vitro (Wang et al., 2006). Isatidis Radix, with an antiviral pharmacological effect, can inhibit a variety of influenza viruses, such as $\mathrm{H} 1 \mathrm{~N} 1, \mathrm{H} 3 \mathrm{~N} 2$, and H9N2, and also has a good anti-inflammatory effect. It can inhibit virus-induced inflammatory response by regulating the NF-kB signaling pathway and the TLR3 signaling pathway (Huang et al., 2019). The antibacterial and antiviral effects of Forsythia suspensa were evaluated by Shi et al. (2013) in combination with the existing in vitro and in vivo studies. The 
study found that Forsythia suspensa has a good inhibitory effect on Escherichia coli, Staphylococcus aureus, Salmonella typhi, Escherichia coli, endotoxin, Staphylococcus epidermidis, influenza A virus, human cytomegalovirus, Japanese encephalitis virus, respiratory syncytial virus, and simple cytomegalovirus. The extract of Agastache rugosa has the ability of immunoregulation. The results showed (Chen et al., 2020d) that the volatile oil of Agastache rugosa leaves had different effects on immune cells at different times. The serum containing Agastache rugosa leaves could significantly activate the white blood cells, macrophages, and lymphocytes of mice and promote the immune function of the body.

\section{MECHANISMS OF TRADITIONAL CHINESE MEDICINE IN THE THERAPY OF CORONAVIRUS DISEASE 2019}

According to the discussion of clinical trials, the above 22 kinds of CPMs have good or potential clinical effects for the prevention and treatment of patients with COVID-19. Therefore, it is particularly important to explore the mechanism of action of CPMs for COVID-19 by computer simulation and in vivo or vitro experimental studies. We reviewed the potential mechanisms of action of CPMs and tried to find more research clues for CPMs with definite curative effect and provide theoretical support for CPMs without clinical research report. The results involving constituents, predicted active ingredients, predicted targets, and regulated signaling pathways for recommended CPMs are summarized in Table 3. The detailed mechanisms are as follows.

\section{Chinese Patent Medicine Has Been Reported in Clinical Trials}

Lianhua Qingwen Capsule has good antiviral, anti-inflammatory, and immunomodulatory effects. Ling et al. (2020) reported that Lianhua Qingwen Capsule could act on SARS-CoV-2 via multiple components, targets, and pathways. Its main ingredients kaempferol, quercetin, and luteolin have better combinations with Mpro, while indigo, glycyrrhetinic acid, and stigmasterol can target the ACE2; thereby, it is possible to cure COVID-19. Li et al (2020b) found that Lianhua Qingwen Capsule could significantly inhibit the replication of SARS-CoV-2 in cells and reduce the expression of viral particles and the upregulation of inflammatory cytokines TNF- $\alpha$, IL6, MCP-1, and IP-10 caused by SARS-CoV-2 infection, in a dose-dependent manner in host cells. It has a comparable antiviral potency against the SARS-CoV-2 with an IC50 value of $411.2 \mu \mathrm{g} / \mathrm{ml}$. Ling et al. (2020), Li et al. (2020b), and Wang et al. (2020c) speculated that Lianhua Qingwen Capsule activity against COVID-19 was exerted through its active ingredients quercetin, luteolin, kaempferol, glycyrrhetinic acid, stigmasterol, and indigo, which influenced inflammatory cytokine targets to regulate the AGE-RAGE, IL17, JAK-STAT, and TNF signaling pathways and other signaling pathways to restrain the viability of SARS-CoV-2 and improve the clinical symptoms of patients with COVID-19. New studies have proved (Li et al., 2019) that Lianhua Qingwen Capsule can effectively reduce the motility chemotaxis potential of macrophages in the acute lung injury (ALI) model and reduce the recruitment of monocyte macrophages by downregulating MCP-1. In vivo, Lianhua Qingwen Capsule can effectively inhibit the infiltration of macrophages, further reduce the edema of lung tissue, reduce the temperature fluctuation caused by ALI, and alleviate the lung pathological injury of ALI.

By using network pharmacology and molecule docking analyses, Gong et al. (2020), Shen et al. (2020), and Jimilihan et al. (2020) identified formononetin, stigmasterol, 3-methoxyglycerol, anhydroicaritin, beta-sitosterol, crude-glycerin, glycyrrhizin B, and other key compounds from Jinhua Qinggan Granules as having a certain degree of affinity with the SARS-CoV-2 3CL hydrolase and ACE2-which might regulate downstream TNF, IL17, PI3K/Akt, and HIF-1 signaling pathways and other signaling pathways and regulate their activity on targets such as PTGS2, HSP90AB1, HSP90AA1, PTGS1, NCOA2, AKTI, and RELA-for the prevention of COVID-19-related lung injury. Mao et al. (2020) and Lin et al. (2020) reported that the mechanism of action of Jinhua Qinggan Granules in the treatment of COVID-19 involved multiple targets and multiple pathways related to antiviral activity, immune regulation, inflammation inhibition, and apoptosis regulation.

Cao et al. (2020) reported that 6-(3-oxoindolin-2-ylidene) indolo(2,1-b)quinazolin-12-one, bicuculline, physciondi glucoside, dihydroverticillatine, and licoisoflavanone are compounds present in Shufeng Jiedu Capsule that could regulate the signaling pathways involved in human cytomegalovirus infection, Kaposi's sarcoma-associated herpesvirus infection, the IL17 signaling pathway, and small cell lung cancer, which could be active to treat COVID-19 by binding with SARS-CoV-2 3CL hydrolase and ACE2. Xu et al. (2020c) speculated that Shufeng Jiedu Capsule might regulate key targets such as IL6, IL1 $\beta$, MAPK8, MAPK1, MAPK14, CCL2, CASP3, FOS, and ALB mainly through flavonoids such as quercetin, luteolin, wogonin, acacetin, isorhamnetin, 5,7,4'trihydroxy-8-methoxyflavone, kaempferol, and phytosterol, which in turn influence the inflammatory response, oxidative stress injury, apoptosis, pulmonary fibrosis, and other processes that reduce lung injury induced by COVID-19. Furthermore, Huang et al. (2020a) and Xu et al. (2020a) reported that Shufeng Jiedu Capsule could treat COVID-19 through multiple components, multiple targets, and multiple pathways of TCM, and its potential mechanism might involve immuneinflammatory response and antiviral activity. Its active ingredients have the potential to help people resist infection by SARS-CoV-2 as they interfere with the binding of SARSCoV-2 to ACE2. Liu et al. (2016) suggested that Shufeng Jiedu Capsule has a significant antipyretic effect, which could reduce the levels of PGE2, TNF- $\alpha$, IL1 $\alpha$, IL6, and IL1 $\beta$, reduce the heat production, and increase the content of AVP.

Also using molecule docking analyses, Deng et al. (2020) found that the core compounds in Huoxiang Zhengqi Oral Liquid such as quercetin, isorhamnetin, irisolidone, kaempferol, wogonin, and baicalein were similar in affinity to currently recommended drugs for the treatment of COVID-19 such as remdesivir, ribavirin, and ritonavir. Of these, isorhamnetin, quercetin, and irisolidone had 


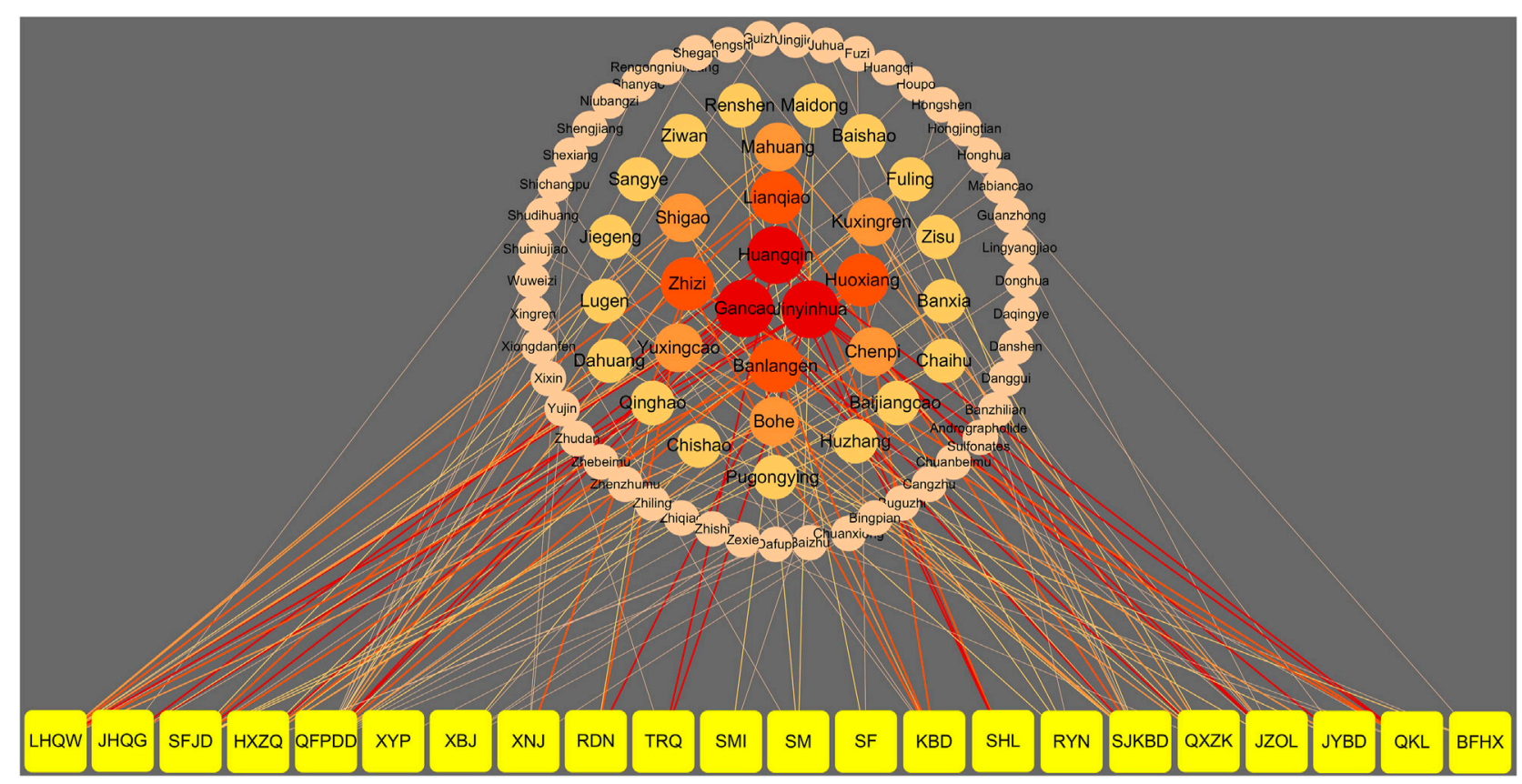

FIGURE 2 | The relationship of the recommended CPMs and their constituents. Note: the yellow nodes represent CPMs. The other color nodes represent constituents of CPMs. The size and darkness of the nodes represent the appearance frequency of each constituent. The darkness of the nodes from dark to light and the size from large to small are illustrated in descending order of appearance frequency. The connecting lines indicate that each node is related. The width of the connecting lines was based on the appearance frequency of constituents, and the color and rule were same as the nodes. LHQW: Lianhua Qingwen Capsule; JHQG: Jinhua Qinggan Granules; SFJD: Shufeng Jiedu Capsule; HXZQ: Huoxiang Zhengqi Capsule; QFPDD: Qingfei Paidu decoction; XYP: Xiyanping Injection; XBJ:Xuebijing Injection; XNJ: Xingnaojing Injection; RDN: Reduning Injection; TRQ: Tanreqing Injection; SMI: Shenmai Injection; SM: Shengmai Injection; SF: Shenfu Injection; KBD: Kangbingdu Granules; SHL: Shuanghuanglian Oral Liquid; RYN: Reyanning Oral Liquid; SJKBD: Siji Kangbingdu Mixture; QXZK: Qingxuan Zhike Granules; JZOL: Jinzhen Oral Liquid; JYBD: Jinye Baidu Granules; QKL: Qingkailing Injection; BFHX: Bufei Huoxue Capsule.

the strongest affinity. These compounds may interfere with ACE2 binding to PTGS2, HSP90AB1, AR, CAMSAP2, and other targets that regulate multiple signaling pathways and thus exert a preventive or therapeutic effect on COVID-19. Du et al. (2020) reported that elicorice glycoside E, naringenin, robinin, [(2R)-7hydroxy-2-(4-hydroxyphenyl)chroman-4-one], and kaempferol strongly bind to $3 \mathrm{CL}$ protease and have been proposed as potential inhibitors of 3CL protease. Furthermore. the antiviral pathway of Huoxiang Zhengqi might be exerted through the PI3KAkt signaling pathway and its downstream effects on viral replication.

Zhao et al. (2021) analyzed the treatment mechanism of Qingfei Paidu decoction from molecular, pathway, and network levels and conducted in vitro experiments to verify. The result showed that the main component of Qingfei Paidu decoction-baicalin-can suppress NF- $\mathrm{BB}$ signaling and downregulate the expression of IL6 and TNF- $\alpha$ and CCL2 in macrophage-like cell line RAQ264.7. In addition, Qingfei Paidu decoction can inhibit the activity of PTGS2 and PGE2 production in order to exhibit the effects of immune regulation and regulate ribosomal proteins related to the viral replication so that it inhibited the growth and production of virus.

In the clinical treatment of patients with COVID-19, Xuebijing Injection mainly inhibited IL6, TNF- $\alpha$, MCP1, mip2, and IL10 to inhibit inflammatory response (Huang et al., 2020a). Xuebijing
Injection could inhibit respiratory viruses while relieving inflammation, delay pathological changes in the lungs, and protect from liver damage caused by viruses or antiviral drugs. It can reduce the apoptosis of tubular cells during sepsis, improve the expression of apoptosis-related proteins Bcl-2 and Bax, and alleviate kidney injury (Sun et al., 2017). It could also inhibit bacterial infections, improve body immunity, and reduce cardiovascular damage (Kang, 2020; Shi X. et al., 2020). Zheng et al. (2020a) reported that hyperoside, salvianolic acid $\mathrm{C}$, salvianolic acid A, cynaroside, salvianolic acid $\mathrm{B}$, and paeoniflorin were the main components of Xuebijing Injection and had a good affinity with SARS-CoV-2 3CL hydrolase and its human receptor, ACE2. Two mechanisms were potentially involved in Xuebijing treatment of SARS-CoV-2-induced pneumonia. First, its main activity could be mainly exerted through the regulation of the human immuneinflammatory response to protect important organs, and second, Xuebijing Injection might also act on the essential proteins of the virus, 3CLpro and ACE2, to induce antiviral effects. Xuebijing Injection presents the characteristics of multiple components and activity against multiple targets and pathways in treating COVID-19, based on its overall synergy (Kong et al., 2020a; He et al., 2020). Chen et al. (2018) showed that $18 \mathrm{ml} / \mathrm{kg}$ Xuebijing Injection could stimulate Treg differentiation and moderately inhibit Th17 differentiation, effectively prevent neutrophil infiltration into lung and kidney, and improve its survival rate in this septic shock model. 
TABLE 3 | Summary of potential action mechanisms of CPMs for COVID-19.

No Name of Chinese patent medicine

1 Lianhua Qingwen Capsule

Ephedra sinica Stapf, Rheum palmatum L., Lonicera Thunb, Menthe canadensis L Glycyrniza glabra L., Rhodiola Glycyrniza glabra L., Ahodiola renclata (Hook.t. \& Thomson) Gypsum Fibrosum, Pogreis.Sm., Gypsum Fibrosum, Pogostemon cablin (Blanco) Benth, Prunus armeniaca L., Houtluynia cordata Thunb, Forsythia suspensa (Thunb. Vahl, Isatis tinctoria L.

2 Jinhua Qinggan Granules Thunb, Artemisia annua L., Arctium lappa L., Scutellaria baicalensis Georgi Fritillaria thunbergii Mia.

3 Shufeng Jiedu Glycyrrhiza glabra L., Forsythia Capsule suspensa (Thunb.) Vahl, Isatis tinctoria L., Reynoutria japonica Houtt, Patrinia scabiosifolia Link, Phragmites australis (Cav.) Trin. Steud., Verbena officinalis $L$ Oral Liquid$$
\text { Bupleurum ch inense DC. }
$$

Glycyrrhiza glabra L., Magnolia officinalis Rehder \& E.H.Wilson, Perilla frutescens (L.) Britton, Areca catechu L., Smilax glabra Roxb, Atractylodes Lancea (Thunb.) DC. Paeonia lactiflora Pall, Citrus $x$ aurantium L., Pinellia ternata (Thunb.) Makino, Pogostemon cablin (Blanco) Benth.

5 Qingfei Paid

Ephedra sinica Stapf, Glycyrrhiza glabra L., Gypsum Fibrosum cutellaria baicalensis Georgi, Bupleurum chinense DC., Smilax glabra Roxb, Citrus $\times$ aurantium $L$. Pogostemon cablin (Blanco) Benth, Neolitsea cassia (L.) Kosterm, Alism plantago-aquatica subsp. orientale Sam, Polyporus umbellatus (Pers.) Fr., Atractylodes macrocephala Koidz, Pinellia ternata (Thunb.) Makino, Zingiber officinale Roscoe, Aster tataricus L.f., Tussilago farfara L., Polygonum aviculare L, Asarum sieboldii Miq Dioscorea oppositifolia L., Prunus armeniaca L.
Chinese name of composition

Mahuang, Dahuang, Jinyinhua, Bohe Gancao, Hongjingtian, Guanzhong.

Shigao, Huoxiang. Kuxingren,

Yuxingcao, Liangiao, Banlangen

PTGS2, IL6, CASP3, MAPK1,

EGFR, ACE2, TNF, IL1B,

MAPK8, CCL2, IL10, IL2,

IFNG, etc.

Qinghao, Jinyinhua, Niubangzi,

Huangqin, Zhebeimu

Huzhang, Baijiangcao, Liangiao

Lugen, Mabiancao, Chaihu

Banlangen, Gancao

Houpo, Banxia, Zisu, Dafupi, Fuling Cangzhu, Baishao, Chenpi, Gancao, Huoxiang

Mahuang, Gancao, Xingren, Shigao, Guizhi, Zexie, zhuling, Baizhu, Fuling Chaihu, Huangqin, Banxia, Shengiiang, Ziwan, Donghua, Shegan, Xixin, Shanyao, Zhishi, Chenpi, Huoxiang

Baicalin, Glycyrrhizic acid, hesperidin, hyperoside

Quercetin, kaempferol, naringenin, isorhamnetin, formononetin, 7-methox 2-methyl, $\beta$-sitosterol, isoflavone, HSP90AB1, HSP90AA1, PTGS1, NCOA2, CALM1, AR,

3'-methoxyglabridin, anhydroicaritin,

stigmasterol, etc.

Quercetin, 6-(3-oxoindolin-2-ylidene) indolo (2,1-b), $\beta$-sitosterol, kaempferol, Iuteolin, quinazolin-12-one, bicuculline (sorhamnetin, physciondiglucoside, 5,7,4'-trihydroxy-8-methoxyflavone acacetin

in, isorhamnetin, irisolidone

kaempferol, wogonin, baicalein

NOS2, ESR1, etc.

PTGS2, ESR1, AR, NOS2, PTGS1, NCOA2, F10, ALB PPARG, PRSS1, SCN5A, IL6, IL1B, FOS, CCL2, MAPK8, MAPK1, MAPK14, CASP3, etc.

PTGS2, HSP9OAB1, AR, CAMSAP2, PPARG, NOS2, etc

TNF signaling pathway, HIFsignaling pathway, toxoplasmosis, bladder cancer, prostate cancer, pancreatic cancer, pathways in cancer

AKT1, TNF- $\alpha$, IL6, PTGS2, HMOX1, IL10, TP53

NOD-like receptor signaling, Toll-like receptor signaling, cy receptor interaction, chemokine signaling, Th17 cell differentiation, L17 signaling pathway, NF-kappa B Zhao et al. (2021) signaling pathway, TNF signaling HIF-1 signaling pathway, mitophagy

A Wang et al., signaling pathway, TNF signaling (2020c); Wang et al. (20200); 
TABLE 3 | (Continued) Summary of potential action mechanisms of CPMs for COVID-19.

\section{No Name of Chines \\ atin name of compositio} patent medicine

Xiyanping Injection

$7 \quad$ Xuebijing Injection Andrographolide sulfonates.

Conioselinum anthriscoides, Carthamus tinctorius L., Angelica sinensis (Oliv.) Diels, Paeonia veitch Lynch, Salvia miltiorrhiza Bunge.

8 Xingnaojing Injection Curcuma aromatica Salisb., Moschus berezovskii Flerov, Dryobalanops aromatica C.F.Gaertn., Gardenia jasminoides J.Ellis.

9 Reduning Injection Lonicera japonica Thunb, Artemisia annua L., Gardenia jasminoides J.Ellis.

10 Tanreaing Injection Lonicera japonica Thunb, Scutellaria baicalensis Georgi, Forsythia suspensa (Thunb.) Vahl, Saig tatarica Linnaeus, Fel Ursi.

11 Shenmai Injection

13 Shenfu Injection

Ophiopogon japonicus (Thunb.) Ker Gawl., Talinum paniculatum (Jacq.) Gaertn.

\section{Ophiopogon japonicus (Thunb.) Ker Maidong, Hongshen, Wuweiz} Gawl., Schisandra chinensis (Turcz) Baill., Talinum paniculatum (Jacq.) Gaertn.

Cyperus rotundus L., Panax ginseng Fuzi, Renshen C.A.Mey.

14 Kangbingdu Granules

Gypsum Fibrosum, Isatis tinctoria L., Z Zhizi, Shichangpu, Banlangen, Pogostemon cablin (Blanco) Benth, Shudihuang, Huoxiang, Shigao Rehmannia glutinosa (Gaertn.) DC Acorus gramineus Aiton, Gardenia jasminoides J.Ellis.

15 Shuanghuanglian Lonicera japonica Thunb, Forsythia Lianqiao, Huangqin, Jinyinhua Oral Liquid suspensa (Thunb.) Vahl, Scutellaria baicalensis Georgi.

\section{Predicted active ingredien}

Andrographolide

Quercetin, gallic acid, luteolin, rosmarinic acid, rutin, kaempferol, chlorogenic acid, tanshinone II A, hydroxysafflor yellow A, paeoniflorin

Chlorogenin, kaempferol

5-Hydroxy-6,7, $3^{\prime}, 4^{\prime}, 5^{\prime}-$

pentamethoxyflavone, isokaempferol,

morin, gardenin, quercetin, artemisetin, genistein, dryobalanone, curcumin elemicin, etc.

Apigenin, quercetin, kaempferol, luteolin, stigmasterol, $\beta$-sitosterol, isorhamnetin , chrysoeriol

Quercetin, baicalein, luteolin, wogonin, kaempferol, scutellarin, baicalin, oroxylin7-O-glucuronide, forsythin, forsythiaside E, ursodeoxycholic acid, chenodeoxycholic acid, etc.

Ophiopogonin D', ophiopogonin D, ginsenoside $r g$ 2, methyl

ophiopogonanone $\mathrm{A}$, ginsenoside $\mathrm{Rb}$ ginsenoside RO, ophiopogon $A$, sanchinoside rd, ophiopogonanone $E$, ginsenoside re, etc

Schisanlactone $\mathrm{E}$ stigmasterol, $\mathrm{N}$-transferuloyltyramine, $\beta$-sitosterol, angeloylgomisin $\mathrm{O}$, gomisin- $\mathrm{A}$, gomisin $\mathrm{A}$, changnanic acid, kadsulactone kadsulignan B, etc. kaempferol, ginsenoside rh2, $\beta$-sitosterol, bisindigotin, stigmasterol, etc.

Angiotensin-converting enzyme 2, bicuculline, luteolin, quercetin, kaempferol, $\beta$-sitosterol, sitosterol, stigmasterol, stigmasterol, irisolidone, 8-isopentenykaempferol, etc.

ercetin, $\beta$-sitosterol, luteolin, stigmasterol, kaempferol, neobaicalein dihydrooroxylin A

\section{Predicted targe}

COX-2, IL6, IL1 $\beta$, TNF, MAPK

MAPK4, MAPK8, MAPK14, etc.

PTGS2, PTGS1, CASP3, RELA,

TNF, MAPK1, IL2, IL6, IL10, etc.

PARP1, PTGS2, MMP9, CDK2,

ADORA2A, ALOX5, GSK3B, etc.

epatitis B, pathways in cancer, TNF signaling pathway, HIF-1 signaling pathway, VEGF signaling pathway, apoptosis

RELA, MAPK1, MAPK14,

MAPK8, IL6, CASP8, CASP3, IL1B, STAT1, TNF, CCL2, etc

TNF, EGFR, NOS3, PTGS2, IL2, GABBR1, MAPK14, ADRB2,

REN, VCAM1, ACHE, PTPRC, IL6, IL1B, MAPK1, IL10, IL4, CXCL8, MAPK14, MAPK8, STAT1, CASPS3, TP53, IFNG

$\mathrm{RB} 1, \mathrm{CXCL2}$, etc.

IL6, GAPDH, ALB, TNF, MAPK1, MAPK3, TP53, EGFR, CASP3, CXCL8

AGE-RAGE signaling pathway, uman cytomegalovirus infection, NF signaling pathway, IL17 signaling pathway

IL17 signaling pathway, T-cel receptor signaling pathway, arachidonic acid metabolic pathway, cAMP signaling pathway, PI3K-AK signaling pathway, influenza A

HIF-1 signaling pathway, TNF signaling pathway, sphingolipid signaling pathway, toll-like signaling pathway, neurotrophin signaling pathway

CASP3, CASP8, PTGS2, BCL2, BAX, PRKCA, PTGS1, PIK3CG, F10, NOS3, DPP4 NOS2, TLR9, ACE, ICAM1, PRKCE, etc.

CASP3, MAPK8, IL1B, PPARG, PTGS2, CASP8, HMOX1, ICAM1, IFNG, NOS2, RELA, STAT1, MAPK14, PPARG,

NOS3, etc

PTGS2, HSP90AB1, PTGS1, TP53, NCOA2, AKT1, JUN, TNF, ESR1, SCN5A, etc.

Prostate cancer, small-cell lung

AGE-RAGE signaling pathway, p53 signaling pathway, small-cell lung cancer, apoptosis

AGE-RAGE signaling pathway, IL17 signaling pathway, C-type letcin receptor, HIF-1 signaling al. (2020); Che et al. $(2020)^{2}$ Kong et al. (2020b) CASP3, TP53, MAPK8, IL6, MAPK1, MAPK1, CCL2, etc. TNF signaling pathway, HIF-1
signaling pathway, pathway in cancer, tuberculosis, hepatitis C, pertussis, salmonella infection, influenza $A$, herpes simplex virus infection 
TABLE 3 | (Continued) Summary of potential action mechanisms of CPMs for COVID-19.

No Name of Chinese Latin name of composition Chinese name of composition patent medicine

Predicted target

Regulated signaling pathways

References

16 Reyanning Oral Reynoutria japonica Houtt, Patrinia Pugongying, Banzhilian, Huzhang, Liquid scabiosifolia Link, Taraxacum Baijiangcao

mongolicum Hand.-Mazz.

Apigenin, chrysin-5-methylether, catechin, $7,2^{\prime}$-dihydroxy-5,8-dime thoxyflavone, 7

hydroxy-5,8-dimethoxy-2-phenyl-

CCL2, CD40LG, CXCL10,

IL17 signaling pathway,

CXCL8, IFNG, IL10, IL13, IL1B, cytokine-cytokine receptor

Wang et al. (2020e)

Scutellaria barbata D. Do

17 Siji Kangbingdu

Mentha canadensis L., Glycyrrhiza glabra L., Prunus armeniaca L,

chromone, 5,7-dihydroxy-8-methoxy-2-

IL2, IL6, etc.

interaction pathway

Mixture

glabra L., Prunus armeniaca L.,

Sangye, Zisu, Juhua, Lugen, Jingjie,

-methoxyphenyl)chromone, etc.

Houttuynia cordata Thunb, Forsythia

suspensa (Thunb.) Vahl, Phragmites

frutescens (L.) Britton, Morus alba L.,

Chrysanthemum $\times$ morifolium

Ramat.) Hemsl., Platycodon

grandiflorus (Jacq.) A.DC., Nepeta

Quercetin, kaempferol, luteolin, rutin,

PTGS1, ADRB2, JUN, IL6, IL1B,

L17 signaling pathway,

Liu et al. (2020b)

Liangiao, Jiegeng

L10, IFNG, ACHE II2, etc.

cytokine-cytokine receptor

interaction, viral protein interaction

with cytokine and cytokine receptor,

rachidonic acid metabolism

cataria L.

18 Qingxuan Zhike

Mentha canadensis L., Glycyrrhiza

glabra L., Prunus armeniaca L.,

Paeonia lactiflora Pall, Citrus $x$

aurantium L., Aster tataricus L.f.,

Morus alba L., Platycodon

19 Jinzhen Oral Liquid $\quad$ Ephedra sinica Stapf, Rheum

palmatum L., Lonicera japonica

Thunb, Scutellaria baicalensis

Georgi, Saiga tatarica Linnaeus,

Lapis Chiloriti, Bovis Calculus Artif Actus, Fritillaria cirrhosa D.Don.

20 Jinye Baidu Granules Lonicera japonica Thunb, Houttuynia cordata Thunb, Taraxacum

Kuxingren, Chenpi, Baishao, Ziwan, Sangye, Gancao, Bohe, Jiegeng. Zhiqiao

Quercetin, kaempferol, luteolin, 7 methoxy-2-methyl, formononetin, etc.

PTGS2, ESR1, HSP9OAA CALM1, AR, etc.

Mengshi, Chuanbeimu, Lingyangiiao, Rengongniuhuang, Huangqiin, Jinyinhua, Dahuang, Mahuang mongolicum Hand.-Mazz. Isats tinctoria L.

21 Qingkailing Injection Lonicera Japonica Thunb, Isatis tinctoria L., Scutellaria baicalensis Georgi, Gardenia jasminoides J.Ellis, Cornu Margaritifera, Cornu Bubali.

22 Bufei Huoxue Capsule mongholicus Bunge, Cullen
Daaingye, Pugongying, Yuxingcao, Jinyinhua

\section{Shuiniujiao, Zhenzhumu, Jinyinhua,} Zhizi, Banlangen, Huangqin

Huangqi, Buguzhi, Chishao
Isoglabrolide, glabrolide, ebeiedinone, desoxo-glabrolid-acetate, peimisine, verticinone, imperialine, ussuriedinone, euchrenone A5, quercetin, kaempfero, naringenin, baicalein, etc.

Kaempferol, glycyrol, indirubin, etc.

Acacetin, syrigin, kaempferol, quercetin, emodin, luteolin, apigenin, etc.

Quercetin, kaempferol, baicalein, 7-Omethylisomucronulatol, formononetin,

$\beta$-sitosterol, stigmasterol, isorhamnetin , ellagic acid, 3,9-di-O-methylnissolin, etc. corylifolium (L.) Medik.

mTOR, JAK3, ACE2, TNF- $\alpha$, AKT2, PIK3CA, MEK1, BRD2, ACE, ANPEP, MAPK3, MAPK ACE, ANPEP, MAPK3, MAPK CXCL8, CCL2, etc.

TNF, ॥1, ॥6, ॥8, PTGS2, PTGS1, NOS3, PPARG, etc.

CASP3, CASP8, FASLG, EGFR, TNF signaling pathway, Fc epsilon Rl, Z Zhang et al. (2020c CYCS, EGF, BCL2L1, IL4, PI3K-Akt signaling pathway

PPARG, MCL1, etc.

IL6, MAPK8, PTGS2, PTGS1, NCOA2, NOS2, RELA, CXCL8,

Pertussis, Chagas disease, TNF L10, MAPK1, FOS, CASP3, gnaling pathway, Salmonella

I3K-Akt signaling pathway, JakSTAT signaling pathway, TNF

Tao et al. (2020) signaling pathway, MAPK signaling pathway infection, tuberculosis

Small cell lung cancer, non-small-cell pathway

IL1A, etc. 
Reyanning Mixture is recommended by the Diagnosis and Treatment Protocol for COVID-19 (trial version 2) statement issued by the Shanxi Province for the therapy of clinically mild and severe cases. Reyanning Mixture exerted beneficial effects in treating coronavirus pneumonia mice by its activity on the lungs. The result showed that Reyanning Mixture can reduce the lung index of coronavirus pneumonia mice with pestilence attacking the lung, significantly increase the percentage of $\mathrm{CD}^{+} \mathrm{T}$ and $\mathrm{CD}^{+} \mathrm{T}$ lymphocytes in peripheral blood of model mice, increase the percentage of total B lymphocytes, reduce virus load in lung tissue, reduce the levels of TNF- $\alpha$, IFN- $\gamma$, IL6, and IL10 in the lung tissue, and reduce the content of motilin in the serum of model mice. It manifested obvious therapeutic effects by improving lung lesions, enhancing gastrointestinal function, improving autoimmune function, and reducing the expression of inflammatory factors in vivo, which provide a rationale for future clinical applications of the active compounds (Bao et al., 2020). Wang et al. (2020e) reported that the core compounds in Reyanning Mixture such as apigenin, chrysin5-methylether, and catechin act on CD40LG, CXCL10, CXCL8, IL10, IL2, IL6, and other targets involved in the IL17 signaling pathway and cytokine-cytokine receptor interaction pathway.

Jinye Baidu Granules are suitable for the treatment of viral pneumonia, acute upper respiratory tract infection, influenza, and other viral and bacterial diseases. It has been used for the prevention and control of SARS virus infection during the SARS outbreak in 2003 and achieved good results. At present, it has been listed as a reserved drug for the prevention and control of COVID-19 in Hubei, Guangdong, Hebei, Fujian, and Anhui Provinces. In the presence of a cytokine storm causing DIC, sepsis, Jinye Baidu Granules particles exert a protective effect on liver microsomal activity, calcium homeostasis, in addition to resisting free radical production and activity, and reducing levels of circulating or tissue inflammatory cytokines (such as TNF- $\alpha$, IL1, IL6, and IL8) and inhibiting dysregulated TNF- $\alpha$ release, which lead to the reduction of vascular endothelial cells and organ damage and prevention of the activation of the blood coagulation system (Shi et al., 2020a).

Pu et al. (2020), Gao et al. (2020), and Chen et al. (2020e) reported that Reduning Injection might act on targets such as inflammatory cytokines and MAPKs through its active ingredients quercetin, kaempferol, beta-sitosterol, luteolin, isorhamnetin, and chrysoeriol, to regulate the AGE-RAGE, human cytomegalovirus infection, TNF, and IL17 signaling pathways and to treat COVID-19 patients. In addition, the antipyretic mechanism of Reduning Injection is related to inflammatory mediators such as IL1, ET-1, IL6, and PGE 2 and regulating endogenous pyrogen (Wang et al., 2013).

Kong et al. (2020b) showed that kaempferol, quercetin, baicalein, luteolin, and wogonin, which are the active compounds of Tanreqing Injection, showed good affinity toward SARS-CoV-2 3CL hydrolase. The molecular mechanism of Tanreqing Injection in the treatment of COVID-19 involved the synergistic features of multiple components, targets, and pathways of TCM.

\section{Chinese Patent Medicines with Potential Therapeutic Effect}

Cai et al. (2020) reported andrographolide, a main component of Xiyanping Injection, had potential antiviral effects in the treatment of COVID-19. It could reduce the level of inflammation in patients, improve respiratory symptoms, inhibit concurrent bacterial infection, and improve the immune response. The research showed (Yang and Luo, 2017) that andrographolide can reduce the level of Th17 and Th2 type cells and play an immunomodulatory role. At the same time, it would not induce similar immunosuppressive effects of steroids, and the incidence of adverse reactions was low. Andrographis paniculata Nees, which has the same active ingredient as Xiyanping Injection, can inhibit LPS-induced hyperthermia in rabbits by reducing the levels of 5-HT and increasing the levels of cAMP in the brain (Xu et al., 2019). In addition, andrographolide can reduce the inflammatory damage of the liver by downregulating $\mathrm{NF}-\kappa \mathrm{B}$ signaling pathway to reduce the expression of inflammatory factors, including TNF- $\alpha$ and IL6.

Xie et al. (2020) discovered the core compounds in Xingnaojing Injection including chlorogenin, 3methylkempferol, kaempferol, morin, 5-hydroxy-6,7,3',4',5'pentamethoxyflavone, gardenin, quercetin, dryobalanone, artemisetin, genistein, curcumin, and elemicin. These compounds might interfere with various signaling pathways by acting on the key targets, such as PARP1, PTGS2, MMP9, CDK2, ADORA2A, ALOX5, and GSK3B. They may also regulate the inflammatory response, apoptosis, oxidative stress, angiogenesis, and the other processes to alleviate the neurological damage sometimes caused by SARS-CoV-2. Further, they inhibited viral replication and prevented infection of the host cell by binding with Mpro, and the ACE2 complex. These activities implied that Xingnaojing Injection might have a positive therapeutic effect on the neurological damage caused by SARS-CoV-2.

Shenfu Injection, Shenmai Injection, and Shengmai Injection are recommended for severe patients, which have a good role in relieving cytokine storm. Their main components are similar, which can inhibit inflammatory factors and alleviate cytokine storm. Han et al. (2020) claimed that ophiopogonin D', ophiopogonin D, ginsenoside Rg2, ophiopogon A, methyl ophiopogonanone A, ophiogenin-3-O-a-L-rhamnopyranosyl, ginsenoside $\mathrm{Rb} 2(1 \rightarrow 2)-\beta$-D-glucopyranoside, ginsenoside $\mathrm{R} 0$, sanchinoside $\mathrm{Rd}$, ophiopogonanone $\mathrm{E}$, and ginsenoside $\mathrm{Re}$ in Shenmai Injection showed a higher binding affinity with $3 \mathrm{CL}$ hydrolase. These compounds were the main effective components in the treatment of COVID-19 combined with coronary heart disease. Shenmai Injection could achieve simultaneous intervention of COVID-19 and coronary heart disease by inhibiting cytokine storms, maintaining cardiac function homeostasis, and regulating immunity and antiviral activity. It exerted a mutual influence and complex interference on a network regulatory mechanism. Fang et al. (2012) reported that Shenmai Injection could cut down the expression of TNF- $\alpha$ and ICAM-1 to play a protective role against lung injury. Li et al. (2020d) reported that kaempferol, ginsenoside Rh2, beta-sitosterol, stigmasterol, and deoxyandrographolide might be the main active ingredients in Shenfu Injection which cause inhibition of the SARS-CoV-2 3CL hydrolase activity and regulate ACE2. As a result, the antiviral effects, immunoregulation, and targeting of the cytokine storm by 
TABLE 4 | The pharmacological action of CPMs for the treatment of COVID-19.

\begin{tabular}{|c|c|c|c|c|c|c|c|}
\hline Category & Name & $\begin{array}{l}\text { Anti-inflammatory } \\
\text { and } \\
\text { immunoregulation }\end{array}$ & Antiviral & $\begin{array}{l}\text { Improving lung } \\
\text { injury }\end{array}$ & Antipyretic & $\begin{array}{l}\text { Organ } \\
\text { protection }\end{array}$ & Reference \\
\hline \multirow[t]{10}{*}{$\begin{array}{l}\text { Clinical efficacy has } \\
\text { been reported }\end{array}$} & $\begin{array}{l}\text { Lianhua Qingwen } \\
\text { Capsule }\end{array}$ & ++ & ++ & ++ & - & - & (Li et al., 2020b); (Li et al., 2019) \\
\hline & $\begin{array}{l}\text { Jinhua Qinggan } \\
\text { Granules }\end{array}$ & ++ & ++ & - & - & - & $\begin{array}{l}\text { (Shen et al., 2020); (Jimilihan } \\
\text { et al., 2020) }\end{array}$ \\
\hline & $\begin{array}{l}\text { Shufeng Jiedu } \\
\text { Capsule }\end{array}$ & ++ & ++ & - & ++ & - & $\begin{array}{l}\text { (Cao et al., 2020); } \\
\text { (Xu et al., 2020c); } \\
\text { (Liu et al., 2016); }\end{array}$ \\
\hline & $\begin{array}{l}\text { Huoxiang Zhengqi } \\
\text { Oral Liquid }\end{array}$ & ++ & ++ & - & - & - & $\begin{array}{l}\text { (Deng et al., 2020); (Du et al., } \\
\text { 2020) }\end{array}$ \\
\hline & $\begin{array}{l}\text { Qingfei Paidu } \\
\text { decoction }\end{array}$ & ++ & ++ & - & - & - & (Zhao et al., 2021) \\
\hline & Xuebijing Injection & ++ & ++ & - & - & ++ & $\begin{array}{l}\text { (Zheng et al., 2020a); (Chen et al., } \\
\text { 2018); (Huang et al., 2020a) }\end{array}$ \\
\hline & $\begin{array}{l}\text { Reyanning Oral } \\
\text { Liquid }\end{array}$ & ++ & - & ++ & - & - & $\begin{array}{l}\text { (Bao et al., 2020); } \\
\text { (Wang et al., 2020e) }\end{array}$ \\
\hline & $\begin{array}{l}\text { Jinye Baidu } \\
\text { Granules }\end{array}$ & ++ & - & - & - & ++ & (Shi et al., 2020a) \\
\hline & Reduning Injection & ++ & - & - & ++ & - & $\begin{array}{l}\text { (Chen et al., 2020e); (Wang et al., } \\
\text { 2013) }\end{array}$ \\
\hline & Tanreqing Injection & ++ & ++ & ++ & - & - & (Kong et al., 2020b) \\
\hline \multirow[t]{12}{*}{$\begin{array}{l}\text { Potential therapeutic } \\
\text { effects }\end{array}$} & Xiyanping Injection & ++ & - & ++ & ++ & ++ & $\begin{array}{l}\text { (Cai et al., 2020); } \\
\text { (Yang and Luo, 2017); (Xu et al., } \\
\text { 2019) }\end{array}$ \\
\hline & $\begin{array}{l}\text { Xingnaojing } \\
\text { Injection }\end{array}$ & ++ & - & - & - & - & (Xie et al., 2020) \\
\hline & Shenmai Injection & ++ & - & ++ & - & ++ & $\begin{array}{l}\text { (Han et al., 2020); } \\
\text { (Fang et al., 2012) }\end{array}$ \\
\hline & Shengmai Injection & ++ & - & ++ & - & - & $\begin{array}{l}\text { (Wang et al., 2020d); (Liu et al., } \\
\text { 2009) }\end{array}$ \\
\hline & Shenfu Injection & ++ & ++ & ++ & - & - & $\begin{array}{l}\text { (Li et al., 2020d); (Wang et al., } \\
\text { 2020b) }\end{array}$ \\
\hline & $\begin{array}{l}\text { Kangbingdu } \\
\text { Granules }\end{array}$ & ++ & ++ & - & - & - & (Yao et al., 2020) \\
\hline & $\begin{array}{l}\text { Shuang Huanglian } \\
\text { Oral Liquid }\end{array}$ & ++ & - & - & ++ & ++ & $\begin{array}{l}\text { (Zheng et al., 2020b); (Wang } \\
\text { et al., 2020f); } \\
\text { (Liang and Zhou, 2011) }\end{array}$ \\
\hline & $\begin{array}{l}\text { Siji Kangbingdu } \\
\text { Mixture }\end{array}$ & ++ & ++ & - & - & - & (Liu et al., 2020b) \\
\hline & $\begin{array}{l}\text { Qingxuan Zhike } \\
\text { Granules }\end{array}$ & ++ & ++ & - & - & - & $\begin{array}{l}\text { (Qiao, 2020); } \\
\text { (Zong et al., 2020) }\end{array}$ \\
\hline & Jinzhen Oral Liquid & ++ & ++ & ++ & ++ & - & $\begin{array}{l}\text { (Tao et al., 2020); (Hong et al., } \\
\text { 2021); (Xiao et al., 2009) }\end{array}$ \\
\hline & Qingkailing Injection & ++ & - & - & ++ & ++ & $\begin{array}{l}\text { (Zhang et al., 2020c); (Gao et al., } \\
\text { 2013); (Sun et al., 2005) }\end{array}$ \\
\hline & $\begin{array}{l}\text { Bufei Huoxue } \\
\text { Capsule }\end{array}$ & ++ & ++ & - & ++ & - & $\begin{array}{l}\text { (Jing et al., 2017); (Guo et al., } \\
\text { 2020a) }\end{array}$ \\
\hline
\end{tabular}

Shenfu decoction may play an important role in the treatment of critically ill patients with COVID-19 through regulating multiple signaling pathways including the AGE-RAGE signaling pathway in diabetic complications, IL17, C-type lectin receptor, and HIF-1 signaling pathways. Shenfu Injection also can reduce the seriousness of lung injury (Wang et al., 2020b). Wang et al. (2020d) found that the active compounds in Shengmai Injection, such as schisanlactone E, N-trans-feruloyltyramine, and stigmasterol, could act on CASP3, PTGS2, NOS2, NOS3, and other targets to regulate multiple signaling pathways to induce anti-inflammatory effect, immune regulation, and antishock and increase blood oxygen saturation in the treatment of COVID-19. Shengmai Injection could reduce the levels of iNOS and NF-kB, improve the peroxidation damage, and has a protective effect on acute lung in rats (Liu et al., 2009).

Kangbingdu Granules had a significant role in combating the SARS virus in 2003 and the influenza A (H1N1) virus in 2009, and thus, the formulation was recommended for pediatric prophylaxis and for adults with fever or cough and sore throat during medical diagnosis and treatment of COVID-19 in Guangdong Province. Yao et al. (2020) reported that the core active compounds such as bicuculline, luteolin, and quercetin in 


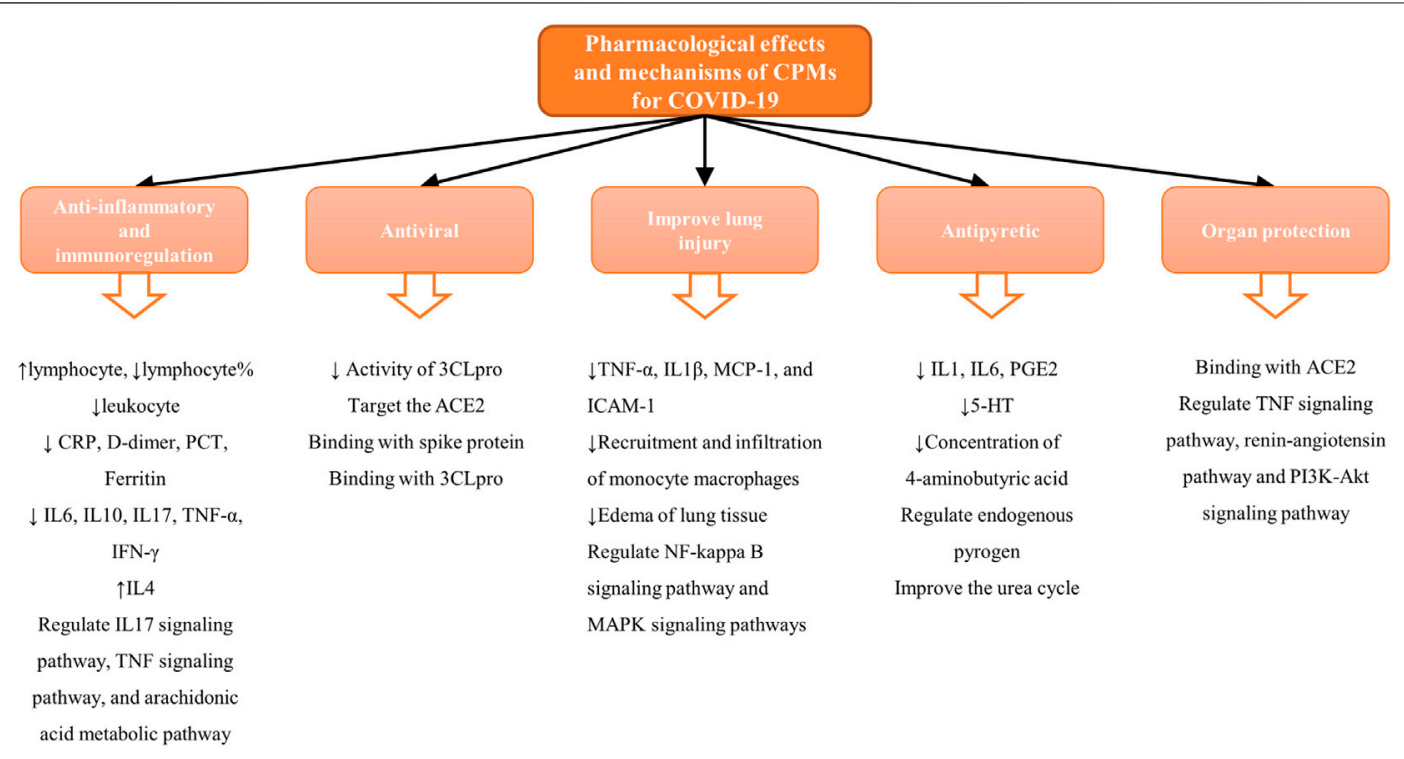

FIGURE 3 | The pharmacological effects and mechanisms of CPMs for COVID-19.

Kangbingdu Granules showed good affinity to SARS-CoV-2 3CL protease. They could interact with ACE2 via its targets PTGS2, HSP90AB1, and PTGS1 to regulate multiple signaling pathways, thereby exerting therapeutic effects on COVID-19.

Shuanghuanglian Oral Liquid is recommended by the Diagnosis and Treatment Protocol for COVID-19 in Beijing. Recent studies have revealed that Shuanghuanglian Oral Liquid has broad-spectrum antiviral and antibacterial activities, which can improve the immune response of the human body. It is an effective broad-spectrum antiviral agent. Zheng et al. (2020b) reported that the mechanism of Shuanghuanglian Oral Liquid against COVID-19 involved its active compounds including quercetin, stigmasterol, beta-sitosterol, kaempferol, neobaicalein, dihydrooroxylin $\mathrm{A}$, and luteolin. These compounds might regulate related biological processes and signaling pathways by acting on key proteins involved in protein network interaction such as CASP3, TP53, MAPK8, IL6, and CCL2. The research showed that Shuanghuanglian Oral Liquid has a protective effect against acute liver failure in mice, which is mainly reflected in improving liver function, inhibiting cytokine synthesis, and reducing inflammatory injury (Wang et al., 2020f). Shuanghuanglian Oral Liquid also can inhibit the LPS-induced increase of body temperature in rats (Liang and Zhou, 2011).

The Siji Kangbingdu Mixture is a CPM recommended by the Diagnosis and Treatment Protocol for COVID-19 (trial version 2) in Shanxi Province and is applicable to mild and severe cases. Liu et al. (2020b) indicated that Siji Kangbingdu Mixture exhibited the potential to be an outstanding agent with better effectiveness and safety compared to chemical antiviral agents and predicted that the Siji Kangbingdu Mixture treatment presented a "win-win" mechanism because it was beneficial not only to symptom alleviation but also for disease resolution. The mechanism might be related to the IL17 signaling pathway and the regulation of the arachidonic acid metabolism pathway which is active in the systemic immune response and inflammation and may also interfere with SARSCoV-2 replication by inhibiting 3CL protease.

Qingxuan Zhike Granule is recommended for COVID-19 patients with mild and moderate disease in Hunan Province, where special TCM treatment protocols for children have been introduced. Experimental studies (Ruan et al., 2010; Qiao, 2020) have shown that Qingxuan Zhike Granule could repair and improve the pathological damage caused by acute bronchitis in model rats and enhanced the bactericidal effect in rat cells. Zong et al. (2020) reported that the core compounds in Qingxuan Zhike Granules including quercetin, kaempferol, and luteolin had a similar affinity with SARS-CoV-2 3CL protease as the recommended drugs. The active ingredients in Qingxuan Zhike Granules might regulate multiple signaling pathways by acting on targets such as PTGS2, HSP90AA1, and ESR1, thereby ameliorating disease progression.

Jinzhen Oral Liquid is mainly used to treat children with bronchitis, bronchopneumonia, mycoplasma pneumonia, and viral pneumonia as well as patients with phlegm-heat cough. At present, Jinzhen Oral Liquid has become an important therapeutic aid. In addition, the academic Zhong Nanshan pointed out that Jinzhen Oral Liquid presented good antiviral and anti-inflammatory activities in vitro at the 24th press conference on COVID-19 prevention and control held by the Information Office of Guangdong Provincial Government. The results of modern pharmacology have shown that Jinzhen Oral Liquid presents good inhibitory or antiviral effects on the SARS virus, influenza A virus, respiratory syncytial virus, and Mycoplasma pneumoniae (Xiao et al., 2008; Xiao et al., 2009). Tao et al. (2020) reported that isoglabrolide, glabrolide, ebeiedinone, desoxo-glabrolid-acetate, peimisine, verticinone, imperialine, ussuriedinone, and euchrenone A5 were the active 
A

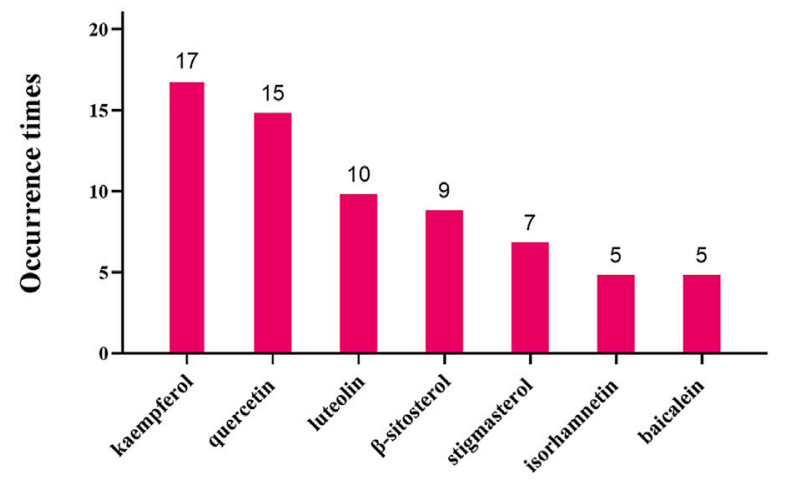

Active ingredients
B

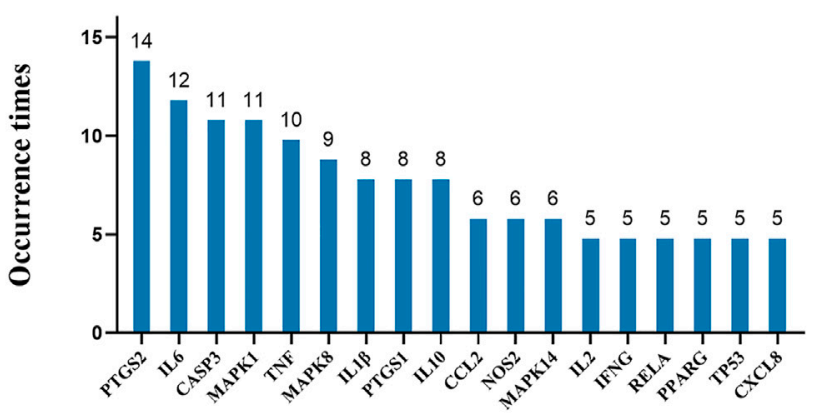

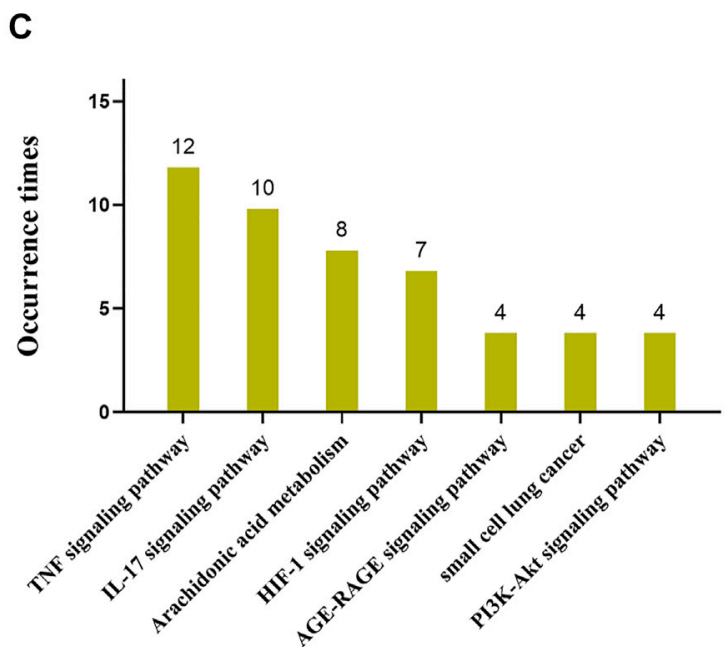

Signaling pathways

FIGURE 4 | Occurrence times of potential mechanism. Note: (A) Occurrence times of active ingredients (frequency $\geq 5$ ). (B) Occurrence times of predicted targets (frequency $\geq 5$ ). (C) Occurrence times of signaling pathways (frequency $\geq 4$ ).

compounds of Jinzhen Oral Liquid in the treatment of COVID19. Jinzhen Oral Liquid might inhibit the occurrence and development of the cytokine storm in COVID-19 by regulating the expression of the bromine domain containing protein 2 (Brd2), aminopeptidase $\mathrm{N}(\mathrm{APN})$, and ACE2, by interfering with the PI3K-Akt, Jak-STAT, TNF, and MAPK signaling pathways, and by inhibiting viral replication by binding with $3 \mathrm{CL}$ protease-thus exerting a preventive or therapeutic effect on COVID-19. In vivo experiments showed that Jinzhen Oral Liquid can alleviate acute lung injury by inhibiting multiple targets and blocking the NF- $\kappa B$ and MAPK signaling pathways (Zong et al., 2018), and it has a good antipyretic effect and is similar to the therapeutic mechanism of Xiyanping Injection (Hong et al., 2021).
Qingkailing Injection is widely used in the treatment of acute pneumonia, upper respiratory tract infection, high fever, and other diseases. Qingkailing Injection has been listed as a recommended drug by the latest version of the Diagnosis and Treatment Protocol for COVID-19 and Prevention Protocol formulated by the Tongji Hospital in Wuhan, Hubei Province. Zhang et al. (2020b) reported that the effects of Qingkailing Injection showed activity against multiple targets and multiple pathways. The active components including acacetin, syrigin, luteolin, kaempferol, quercetin, and apigenin could regulate the apoptosis pathway and the TNF pathway by acting on CASP3, CASP8, FASLG, and other targets, to achieve potential therapeutic effects on COVID-19. Gao et al. (2013) suggested that Qingkailing Injection has an antipyretic effect by decreasing the 
expression of 5-HT and the concentration of 4-aminobutyric acid and it can improve the metabolism of amino acids and the urea cycle. Sun et al. (2005) used LPS to cause visceral injury, and the results reflected that Qingkailing Injection had the function of protecting visceral injury as reflected through enhancing the freeradical activity and the inhibition to overoxidation by the results of detecting MDA and SOD levels.

Recent studies have suggested that the aggravation and even deaths caused by viral diseases such as COVID-19 were not only related to pulmonary virus infection, but were also closely associated with the immune dysfunction of the body (Xu et al., 2020d). The studies have shown that Bufei Huoxue Capsule could effectively improve pulmonary fibrosis, reduce the expression of inflammatory factors such as TNF- $\alpha$ and IL6, promote lung tissue repair, and effectively improve the immune response, which also contributes to the recovery of COVID-19 (Ma et al., 2016; Jing et al., 2017). Currently, Bufei Huoxue Capsule has been included in the recommended drug list in the Diagnosis and Treatment Protocol for COVID-19 in Guizhou Province and is mainly used for COVID-19 recovery treatment. Guo et al. (2020a) reported that the beneficial effects of Bufei Huoxue Capsule were not only associated with its antiviral and anti-inflammatory activities, but were also closely related to the regulation of immune function in the treatment of convalescent COVID-19 patients. Its key compounds including quercetin, kaempferol, 7-O-methylisomucronulatol, baicalein, and formononetin target IL6, MAPK8, PTGS2, PTGS1, and NCOA2 to regulate multiple signal pathways of TCM and play a therapeutic role in the recovery period of COVID-19.

\section{Common Pharmacological Actions and Mechanisms}

On the basis of reviews and comments on the previous text, we concluded that these 22 recommended CPMs in the treatment of COVID-19 mainly act on anti-inflammatory and immunoreguation and antiviral effects and improve lung injury, antipyretic effect, and organ protection. The results are provided in Table 4 and Figure 3. In addition, we calculated the occurrence times of active ingredients, predicted targets, and signaling pathways predicted by network pharmacology and molecular docking. The results are shown in Figure 4.

Table 4 shows 22 CPMs which all have anti-inflammatory and immunomodulatory effects. Recent studies have suggested that the pathogenic mechanism of SARS-CoV-2 is closed related to inflammatory stimulation and immune dysfunction (Xu et al., 2020e). The immune system responds rapidly after SARS-CoV-2 has infected the body. $\mathrm{CD}^{+} \mathrm{T}$ lymphocytes differentiate rapidly into helper $\mathrm{T}$ cells $\mathrm{Th} 1$ and Th2, and then, Th1 secretes granulocyte-macrophage colony-stimulating factor (GM-CSF). These cytokines will induce excessive activation of $\mathrm{CD}_{14}{ }^{+}$and $\mathrm{CD}^{+} 6^{+}$monocytes (Zhou et al., 2020b). When the Th1 and Th2 cells and the $\mathrm{CD} 14^{+}$and $\mathrm{CD} 16^{+}$monocytes enter the pulmonary circulation, a significant inflammatory cell infiltration, with the release of proinflammatory cytokines, chemokines, IL1 $\beta$, IL6, IFN- $\gamma$, TNF- $\alpha$, CCL2, CCL3, and CXCL10, will occur in the lungs, forming a "cytokine storm" causing severe pneumonia and in turn lead to lung injury, acute respiratory distress syndrome (ARDS), and multiple organ failure and even death (Huang et al., 2020a; Zhou Y. X. et al., 2020). The regulatory function of TCM on the immune system is closely related to the production and activity of T cells, B cells, NK cells, macrophages, and cytokines. From the results of COVID-19 clinical research, we can find CPMs can regulate the proportion of lymphocyte and leukocyte, significantly reduce the level of inflammation factors such as CRP, D-dimer, PCT, IL6, IL10, IL17, TNF- $\alpha$, and IFN- $\gamma$, and increase the level of IL4 (Chen et al., 2020c; Qin et al., 2020). Network pharmacology research shows that the mechanism may be related to the IL17 signaling pathway, TNF signaling pathway, and arachidonic acid metabolism pathway. The biological activity of TNF is to promote inflammation, cell survival, and tissue regeneration. TNF can effectively stimulate a large amount of IgG, promote the proliferation of $B$ cells, and activate the adhesion receptors on the surface of endothelial cells and neutrophils (Shen et al., 2020). IL17 is a proinflammatory cytokine mainly secreted by Th17 and other T cells, which exerts a major role in adaptive immunity. The IL17 signaling pathway can reduce the aggregation of macrophages and granulocytes by regulating CXCL2, CCL2, CXCL8, and other chemokines, thus reducing the inflammatory response (Noack and Miossec, 2017; Liu Y. R. et al., 2020). Ren et al. (2020c) concluded that arachidonic acid (AA) metabolism pathway is principally used to synthesize inflammatory cytokines, such as interleukin (IL), interferon (IFN), C-X-C motif chemokine (CXCL), and so on, which directly leads to the cytokine cascade reaction. Therefore, the inhibition of the AA metabolism pathway is beneficial for alleviating the "cytokine storm."

The action mechanism of antiviral drugs is mainly through blocking a certain stage of virus replication and reproduction, such as preventing the virus from entering the host cell (Pawlos et al., 2021), inhibiting the synthesis of the virus (Kumar et al., 2021), and inhibiting the release of the virus (Martin-Sancho et al., 2021), so as to achieve the purpose of inhibiting virus reproduction and controlling virus infection. The antiviral effect of CPMs is through inhibiting the function enzyme of SARS-CoV-2 or binding with the host cell receptor. In the second part of this article, we mentioned that the main targets of anti-SARS-CoV-2 include ACE2 and 3CLpro. ACE2 plays an important role in the process of binding with the virus, while 3CLpro is related to viral transcription and replication. CPMs' main ingredients kaempferol, quercetin, luteolin, and baicalein have better combinations with Mpro, while isorhamnetin, stigmasterol, and $\beta$-sitosterol can target the ACE2 (Ling et al., 2020; Kong et al., 2020b; Li Y. M. et al., 2020). Kaempferol glycosides are good candidates for 3a channel proteins of coronaviruses (Schwarz et al., 2014). Quercetin was proven to exert an inhibitory effect on PEDV 3CLpro (Li et al., 2020e) and interfere with multiple steps of pathogen virulence viral entry, viral replication, and protein assembly and these therapeutic effects can be enhanced by the $\mathrm{CO}$ administration of vitamin $\mathrm{C}$. quercetin (Colunga et al., 2020). Luteolin has been reported in experimental studies to inhibit viral replication by binding to the spike protein on the surface of the coronavirus and can inhibit 3CLpro, which is required for viral infection (Ling et al., 2004). Liu 
et al. (2021) found that baicalein could inhibit SARS-CoV-2 3CLpro activity in vitro with IC50 values of $8.52 \mu \mathrm{g} / \mathrm{ml}$ and $0.39 \mu \mathrm{M}$. The effect of isorhamnetin binding to ACE2 was demonstrated by the surface plasmon resonance assay, and it was shown by in vitro virus experiments that isorhamnetin could inhibit SARS-CoV-2 spike pseudotyped virus entering ACE2h cells (Zhan et al., 2021). Antiinflammatory and antiviral effects of stigmasterol and $\beta$-sitosterol were demonstrated in in vitro experimental studies (Petrera et al., 2014; Liao et al., 2018). In addition, kaempferol, quercetin, luteolin, baicalein, and isorhamnetin all belong to flavonoids. Studies have shown that flavonoids could interact with the S1 and S2 sites of MERS-CoV 3CLpro to play a role in antiviral effects (Jo et al., 2019; Ren et al., 2020b).

The SARS-CoV-2 infects humans mainly through the ACE2 receptor and the main invasion site is AT2 cells, resulting in severe lung injury (Kuba et al., 2005). Histopathological changes were also most common in the lungs and blood vessels. The mechanism of lung injury is mainly constituted by two aspects, the production of a variety of inflammatory cytokines by alveolar macrophages and lung epithelial cell activation, such as IL1 $\beta$, IL2, IL6, IL7, IL8, IL10, tumor necrosis factor- $\alpha$ (TNF- $\alpha$ ), granulocyte-macrophage colonystimulating factor (GM-CSF), and interferon-gamma-induced protein 10 (IP-10) (Zarrilli G et al., 2021). On the other hand, upregulation of endothelial adhesion factor (VCAM, ICAM, VWF, ANG-2, and VEGF) also contributes to lung injury (Polidoro et al., 2020). Treatment of CPMs for COVID-19 is to alleviate excessive inflammatory response in lung tissue mainly by inhibiting the synthesis and release of inflammatory factors. According to the clinical and statistical data, CPMs could cut down the expression of TNF- $\alpha$, IL1 $\beta$, MCP-1, and ICAM-1, reduce the recruitment and infiltration of monocyte macrophages, and further reduce the edema of lung tissue to improve lung injury (Fang et al., 2012; Li et al., 2019). In addition, Jinzhen Oral Liquid can block the NF- $\kappa B$ and MAPK signaling pathways to improve lung injury (Zong et al., 2018).

Fever is considered to be one of the main clinical features and judgments of patients with COVID-19. After fever, immune cells activate and release pyrogenic cytokines IL1, IL6, and TNF- $\alpha$, which then act on essential mediators COX-2 and PGE2 to cause hyperthermia (Ma et al., 2021). The antipyretic mechanism of CPMs is mainly achieved by reducing the cAMP content in hypothalamus and cerebrospinal fluid, inhibiting the synthesis of PGE2, and reducing the content of central neurotransmitter (Xu et al., 2019). Shufeng Jiedu Capsule and Reduning Injection exert an antipyretic effect through regulating the expression of IL1, IL6, and PGE2 and regulate endogenous pyrogen. The mechanism of antipyretic of Qingkailing Injection is to decrease the expression of 5-HT and the concentration of 4-aminobutyric acid and it can improve the metabolism of amino acids and the urea cycle (Gao et al., 2013). Baicalein, an active ingredient with high frequency in TCM, has a good antipyretic effect. Its mechanism of action is to inhibit arachidonic acid metabolism, COX and LOX, and the secretion and release of nuclear factor and cytokine (Xin, 2013).

Although the novel coronavirus pneumonia is a kind of infectious disease directed at the lungs, studies have shown that it can also damage other organs, such as the heart, nerves, brain, vessels, kidneys, and skin (Hossain et al., 2020). SARS-CoV-2 combined with ACE2 stimulated macrophages and monocytes to release proinflammatory cytokines, including IL6, NF-kB, and TNF- $\alpha$, leading to inflammation-derived injurious cascades and then to multiple organ failure. Among them, ACE2 plays a key role in inflammation, which may aggravate COVID-19 through the following ways: renin-angiotensin system, including promoting the pathological changes in lung injury and involving inflammatory response (Iwasaki et al., 2021). In addition, its severity is related to the viral load and the regulation of ACE2 receptor (Loganathan et al., 2021). CPMs show high binding affinity with ACE2 playing a role in organ protection, and many active ingredients can repair organ damage. Kaempferol has a protective effect on oxidative stress-induced nerve injury and hepatocyte apoptosis by inhibiting the expression of apoptotic protein (Schwarz et al., 2014). In vitro test showed that baicalein had cardiovascular protective effects such as vasodilation, hypotension, myocardial protection, endothelial cell protection, and antiatherosclerosis (Xin, 2013). The TNF signaling pathway plays a role in the induction of tissue repairs, such as neuronal remyelination, cardiac remodeling, or cartilage regeneration (Noack and Miossec, 2017). The PI3K-Akt signaling pathway has a protective effect on oxidative stress and inflammatory response induced by various pharmacological media, especially playing a key role in the survival, proliferation, and apoptosis of cardiac myocytes (Li et al., 2021).

\section{CONCLUSION AND FUTURE PROSPECTS}

Among the many plagues experienced in Chinese history, TCM has played an important role in both early prevention and treatment to control the progression of the disease and improve patient prognosis (Yang, 2020b; Ren J.-l. et al., 2020). Currently, the focus and difficulties in the treatment of COVID-19 concern mainly the more severe and critical cases, which are also the main factors directly affecting mortality. The major purpose of the participation of TCM in the therapy of COVID-19 is to make full use of its advantages in the treatment of mild and moderate COVID-19 patients. For severe or critical COVID-19 patients, TCM can reduce the disease condition and decrease mortality (Yang, 2020b). However, vulnerable patient groups such as the elderly, pregnant and lactating women, infants, and patients with underlying diseases need to be especially cautious when using CPMs. Improper usage of CPMs, such as abuse or misuse, repeated drug use, overuse, drug antagonism, drug incompatibility, drug-drug interactions, and improper syndrome classification, will increase drug-induced risks (Zhang et al., 2020c); for example, when there is a repeated incompatibility in early treatment (such as taking Lianhua Qingwen Capsule and Shuanghuanglian oral liquid simultaneously), this incompatibility will influence the effects of treatment. In addition, for drugs containing ephedra (such as Lianhua Qingwen Capsule and Jinghua Qinggan Granules), doctors should pay attention to the patient's blood pressure and heart rate.

In vivo experiments showed (Zhang et al., 2021) that Qingfei Paidu decoction can affect the pharmacokinetics of CYP3A substrate drugs through the inactivation of CYP3A, which leads to the risk of disease treatment with CYP3A substrate drugs such as lopinavir. 
Moreover, severe COVID-19 patients are prone to experience septic shock, liver and kidney dysfunction, and related underlying diseases which will reduce drug metabolism and clearance rates. For these patients, treatment regimens should avoid the use of drugs with the potential to induce liver and kidney toxicity to avoid the possibility of drug accumulation (Yang, 2020b). At the same time, it should also be noted that respiratory viral diseases, including COVID-19, may present symptoms of critical illness. Although there are corresponding treatment methods among TCM, these still present certain limitations. For these reasons, further exploration of TCM treatment of these diseases and syndromes is needed and we should actively cooperate with Western rescue strategies to improve cure rates and reduce death rates.

All of these 22 TCMs we reviewed are currently widely used clinically, and 10 have been reported in the literature to significantly improve clinical symptoms in patients with COVID-19, and a part of them have also been studied. However, due to the low number of COVID-19 cases in China and the lack of a large number of research subjects, it makes clinical research difficult. We provide a comprehensive analysis of the therapeutic effects and mechanisms of the above Chinese patent medicines, while exploring the mechanism of drug treatment, to provide some basis and clues to the clinical therapeutic effects. But this review has some limitations. At present, understanding of the mechanisms involved in TCM for the treatment of COVID-19 has been mainly achieved through virtual simulations and analyses of potential pharmacological networks and molecular docking studies. The limitations of the network pharmacology and molecular docking screening results are also in function of the

\section{REFERENCES}

Bao, L., Shi, Y. J., Geng, Z. H., Sun, J., Zhao, R. H., Du, C. Q., et al. (2020). [Application of Reyanning Mixture in Evaluating Combining Disease with Syndrome of Human Coronavirus Pneumonia with Pestilence Attacking Lung Syndrome]. Zhongguo Zhong Yao Za Zhi 45, 1465-1472. doi:10.19540/ j.cnki.cjcmm.20200303.401

Cai, N., Li, Y. J., Zhou, G. R., Xin, C., Xie, J., Zhou, H. H., et al. (2020). Theoretical Basis and Effect Characteristics of Andrographolide against COVID-19. Chin. Tradit. Herb. Drugs 51, 1159-1166. doi:10.7501/j.issn.02532670.2020.05.01010.1161/strokeaha.119.028010

Cao, C., Cui, Y., Chu, Y. X., Shi, Y. Y., Wu, X. H., Wang, X. Y., et al. (2020). Investigation on Mechanism and Active Components of Shufeng Jiedu Capsule in Treatment of COVID-19 Based on Network Pharmacology and Molecular Docking. Chin. Tradit. Herb. Drugs 51, 2283-2296. doi:10.7501/j.issn.02532670.2020.09.002

Centers for Disease Control and Prevention (2020). Initial Public Health Response and Interim Clinical Guidance for the 2019 Novel Coronavirus OutbreakUnited States, December 31, 2019-February 4, 2020. Available at: http://dc.gov/ mmwr/volumes/69/wr/mm6905e1.htm?s_cid=mm6905e1_w (Accessed February 7, 2020)

Chan, J. F.-W., Yuan, S., Kok, K.-H., To, K. K.-W., Chu, H., Yang, J., et al. (2020). A Familial Cluster of Pneumonia Associated with the 2019 Novel Coronavirus Indicating Person-To-Person Transmission: a Study of a Family Cluster. The Lancet 395, 514-523. doi:10.1016/S0140-6736(20)30154-9

Chen, J., Wang, W. Q., Shi, C. Y., and Fang, J. G. (2020a). Thoughts on Prevention and Treatment of Coronavirus Disease 2019 (COVID-19) by Traditional Chinese Medicine. Chin. Tradit. Herb. Drugs 51, 1106-1112. doi:10.7501/ j.issn.0253-2670.2020.05.003 complexity of TCM and of its compound composition as well as the complexity and variability of the virus. Thus, studies of composition, predicted targets, and pathways may have some limitations. Further in vivo and in vitro experiments should be carried out to verify the validation of these mechanisms to provide a scientific basis for the TCM treatment of COVID-19 and to provide an experimental basis for antiviral natural medicine research and development.

\section{AUTHOR CONTRIBUTIONS}

Z-HY, BW, QM, LW, Y-XL, H-FY, Z-XF, H-JC, ZG, H-JW, and $\mathrm{B}-\mathrm{NZ}$ performed the literature search, selected relevant articles, interpreted data, and wrote the report; Z-HY and BW have contributed equally to this work and share first authorship; FZ was responsible for the entire manuscript; H-DS and Feng L-M designed and supervised this work. All authors have read and approved the final submission.

\section{FUNDING}

This study was supported by the Hebei North University Youth Fund Project (No. JYT2019010), Tianjin University of Traditional Chinese Medicine Doctoral Student Innovation Program Project (No. ZXYCXLX2019016), and Tianjin Health Commission Integrated Traditional Chinese and Western Medicine Project (No. 2019130).

Chen, L., Ge, G. B., Rong, Y., Fu, W., Zheng, M. Y., Zhao, Y. F., et al. (2020b) Application and Research Progress of Traditional Chinese Medicine in Prevention and Treatment of corona Virus Disease 2019. Acta Univ. Tradit. Med. Sin. Pharmcol. Shanghai. 34 (3), 1-8. doi:10.16306/j.1008861x.2020.03.00110.4103/wjtcm.wjtcm_30_19

Chen, L., Liu, F., Wu, J. H., Song, H. Y., Xia, J. S., Sheng, B., et al. (2020c). Clinical Efficacy of Shufeng Jiedu Capsule Combined with Western Medicine in Treatment of Common COVID-19 Patients by Retrospective Analysis. Chin. J. Exp. Tradit. Med. Form., 1-8. doi:10.13422/j.cnki.syfjx.20201628

Chen, R., Wang, T. T., Li, K. L., Shang, R. F., Song, J., and Zhang, J. Q. (2020d). Characteristics and Application of Immune-Regulating and Antiviral Chinese Materia Medica. Chin. Tradit. Herb. Drugs 51 (6), 1412-1426. doi:10.7501/ j.issn.0253-2670.2020.06.006

Chen, X., Feng, Y., Shen, X., Pan, G., Fan, G., Gao, X., et al. (2018). Anti-sepsis protection of Xuebijing Injection Is Mediated by Differential Regulation of Proand Anti-inflammatory Th17 and T Regulatory Cells in a Murine Model of Polymicrobial Sepsis. J. Ethnopharmacology 211, 358-365. doi:10.1016/ j.jep.2017.10.001

Chen, Y. K., Zeng, A., Luo, Z. H., He, S. M., Li, C. M., and Lu, Q. (2020e). Study on the Active Compounds and Potential Mechanism of Reduning Injection in Treating Novel Coronavirus Pneumonia. J. Guangdong Pharm. Univ. 36, 381-387.

Chen, Y. W., Yiu, C.-P. B., and Wong, K.-Y. (2020f). Prediction of the SARS-CoV-2 (2019-nCoV) 3C-like Protease (3CLpro) Structure: Virtual Screening Reveals Velpatasvir, Ledipasvir, and Other Drug Repurposing Candidates. F1000Res 9, 129. doi:10.12688/f1000research.22457.2

Cheng, D. Z., Wang, W. J., Li, Y., Wu, X. D., Zhou, B., and Song, Q. Y. (2020). Analysis of Curative Effect of 51 Patients with Novel Coronavirus Pneumonia Treated with Chinese Medicine Lianhua Qingwen: a Multicentre Retrospective Study. Tianjin. J. Tradit. Chin. Med. 37 (5), 509-516. doi:10.11656/j.issn.16721519.2020.05.06 
Colunga Biancatelli, R. M. L., Berrill, M., Catravas, J. D., and Marik, P. E. (2020). Quercetin and Vitamin C: An Experimental, Synergistic Therapy for the Prevention and Treatment of SARS-CoV-2 Related Disease (COVID-19). Front. Immunol. 11, 1451. doi:10.3389/fimmu.2020.01451

Coronaviridae Study Group of the International Committee on Taxonomy of Viruses (2020). The Species Severe Acute Respiratory Syndrome-Related Coronavirus: Classifying 2019-nCoV and Naming it SARS-CoV-2. Nat. Microbiol. 5 (4), 536-544. doi:10.1038/s41564-020-0695-Z

Deng, Y. J., Liu, B. W., He, Z. X., Liu, T., Zheng, R. L., Yang, A. D., et al. (2020). Study on Active Compounds from Huoxiang Zhengqi Oral Liquid for Prevention of Coronavirus Disease 2019 (COVID-19) Based on Network Pharmacology and Molecular Docking. Chin. Tradit. Herb. Drugs 51, 1113-1122. doi:10.7501/j.issn.0253-2670.2020.05.004

Du, H. T., Wang, P., Ma, Q. Y., Li, N., Ding, J., Sun, T. F., et al. (2020). Preliminary Study on the Effective Components and Mechanism of Huoxiang Zhengqi Decoction in Inhibiting the Replication of Novel Coronavirus. Modernization. Tradit. Chin. Med. Mater. Mater-world. Sci. Tech., 1-7. doi:10.11842/ wst.20200221002

Du, L. N., Yang, Y., Mu, Q. H., Yuan, B., Zhang, J. H., Zhao, Y., et al. (2021). Clinical Study of Qingxuan Zhike Granules in the Treatment of Children with Cold of Wind-Heat Syndrome. Chin. J. Tradit. Chin. Med. Pharm. 36 (02), 1157-1160.

Duan, C., Xia, W. G., Zheng, C. J., Sun, G. B., Li, Z. L., Li, Q. L., et al. (2020). Clinical Observation of Jinhua Qinggan Granule in Treatment of COVID-19. J. Tradit. Chin. Med. 1-5.

Fang, Q., Qu, X. Q., Li, Y. N., Cao, H. L., Gao, Y. J., and Zhang, Z. G. (2012). The Observation of Healing Effect of Rats with Acute Lung Injury Treated with Shenmai Injection. Chin. Anim. Hus. Vet. Med. 39 (1), 101-104. doi:10.3969/ j.issn.1671-7236.2012.01.025

Gallagher, T. M., and Buchmeier, M. J. (2001). Coronavirus Spike Proteins in Viral Entry and Pathogenesis. Virology 279 (2), 371-374. doi:10.1006/viro.2000.0757

Gao, X., Guo, M., Peng, L., Zhao, B., Su, J., Liu, H., et al. (2013). UPLC Q-TOF/MSBased Metabolic Profiling of Urine Reveals the Novel Antipyretic Mechanisms of Qingkailing Injection in a Rat Model of Yeast-Induced Pyrexia. EvidenceBased Complement. Altern. Med. 2013, 1-8. doi:10.1155/2013/864747

Gao, Y., Ming, L., Yang, G. L., Shi, S. Z., Liu, Y. J., Sun, Y., et al. (2020). Preliminary Study on the Effective Components and Mechanism of Reduning Injection in the Treatment of Corona Virus Disease 2019. Chin. J. Med. Guide 22, 145-152.

Gong, P. Y., Guo, Y. J., Li, X. P., Li, N., and Gu, J. (2020). Exploring Active Compounds of Jinhua Qinggan Granules for Prevention of COVID-19 Based on Network Pharmacology and Molecular Docking. Chin. Tradit. Herb. Drugs 51, 1685-1693. doi:10.7501/j.issn.0253-2670.2020.07.002

Guo, S., Wu, W. X., Xie, H., Li, Q., Wang, H. B., and Duang, J. A. (2020a). Molecular Mechanism of Bufei Huoxue Capsule on COVID-2019 Based on Network Pharmacology and Molecular Docking. Chin. Tradit. Herb. Drugs 51, 2307-2316. doi:10.7501/j.issn.0253-2670.2020.09.004

Guo, Y.-R., Cao, Q.-D., Hong, Z.-S., Tan, Y.-Y., Chen, S.-D., Jin, H.-J., et al. (2020b). The Origin, Transmission and Clinical Therapies on Coronavirus Disease 2019 (COVID-19) Outbreak - an Update on the Status. Mil. Med Res 7 (1), 11. doi:10.1186/s40779-020-00240-0

Han, L. W., Zhang, Y. G., Li, H. N., Wang, H. Y., Li, X. B., Wang, X. J., et al. (2020). Network Pharmacologic Molecular Mechanism of Shenmai Injection in Treatment of COVID-19 Combined with Coronary Heart Disease. Chin. Tradit. Herb. Drugs 51, 2334-2344. doi:10.7501/j.issn.0253-2670.2020.09.007

He, T. M., Duan, C. C., Li, X. F., and Zhang, J. Z. (2020). Potential Mechanism of Xuebijing Injection in Treatment of Coronavirus Pneumonia Based on Network Pharmacology and Molecular Docking. Chin. J. Mod. Appl. Pharm. 37, 398-405. doi:10.13748/j.cnki.issn1007-7693.2020.04.004

Hong, Q., Chen, C. X., Liu, Y. X., Wang, Y., and Li, Y. L. (2021). Animal Experimental Study on Antipyretic Mechanism of Jinzhen Oral Liquid. J. Pediatr. Pharm. 27 (2), 1-4. doi:10.13407/j.cnki.jpp.1672-108X.2021.02.001

Hossain, M. F., Hasana, S., Mamun, A. A., Uddin, M. S., Wahed, M. I. I., Sarker, S., et al. (2020). COVID-19 Outbreak: Pathogenesis, Current Therapies, and Potentials for Future Management. Front. Pharmacol. 11, 563478. doi:10.3389/fphar.2020.563478

$\mathrm{Hu}, \mathrm{X}$., and Zhang, L. (2020). Latest Research Progress of Clinical Trials on New Drugs for corona Virus Disease 2019. Clin. Med. J. 18 (3), 11-15. doi:10.3969/ j.issn.1672-3384.2020.03.003
Huang, C., Wang, Y., Li, X., Ren, L., Zhao, J., Hu, Y., et al. (2020). Clinical Features of Patients Infected with 2019 Novel Coronavirus in Wuhan, China. The Lancet 395 (10223), 497-506. doi:10.1016/S0140-6736(20)30183-5

Huang, J., Li, X. W., Lan, B. Z., Li, J. P., You, D. M., and Zhou, S. (2020a). Clinical Observation on Tanreqing Injection Combined with $a$-interferon in the Treatment of Confirmed and Suspected Cases of COVID-19. Chin. J. Misdiagn 15 (09), 408-409.

Huang, J. W., An, L. F., Han, X., Li, Q. W., Xue, H., Yang, L., et al. (2020b). The Potential Mechanism of Prevention and Treatment of COVID-19 by Shufeng Jiedu Capsule Was Studied Based on Network Pharmacology. J. Hainan Med. Univ. 26, 814-819. doi:10.13210/j.cnki.jhmu.20200512.003

Huang, Y., Li, J., Xu, K. Y., Wang, D. Q., and Yang, Z. F. (2019). Research Progress on Anti-influenza Effective Components of Isatidis Radix. Chin. J. Mod. Appl. Pharm. 36 (20), 2618-2623. doi:10.13748/j.cnki.issn1007-7693.2019.20.027

Iwasaki, M., Saito, J., Zhao, H., Sakamoto, A., Hirota, K., and Ma, D. (2021). Inflammation Triggered by SARS-CoV-2 and ACE2 Augment Drives Multiple Organ Failure of Severe COVID-19: Molecular Mechanisms and Implications. Inflammation 44 (1), 13-34. doi:10.1007/s10753-020-01337-3

Jimilihan, S. M. Y., Maimaitiming, N. E. M. M. T., Ainiwaer, W. M. E., Maierdan, Y. S. F., Muhadaisi, N. E., Nuerbiya, N. H. M. T., et al. (2020). Study on the Active Components in the Adjuvant Treatment of Novel Coronavirus Pneumonia (COVID-19) with Jinhua Qinggan Granules Based on Network Pharmacology and Molecular. J. Chin. Med. Mater. 43, 1275-1283. doi:10.13863/j.issn1001-4454.2020.05.046

Jing, Y., Tang, S. H., Jin, J., Cao, R. Q., Wang, Y. Q., Liao, Q., et al. (2017). Influence of Pulmonary Fibrosis in PM2.5 Model Mice with Bufei Huoxue Capsule. World J. Integ. Tradit. West. Med. 12, 774-778.

Jo, S., Kim, H., Kim, S., Shin, D. H., and Kim, M. S. (2019). Characteristics of Flavonoids as Potent MERS-CoV 3C-like Protease Inhibitors. Chem. Biol. Drug Des. 94 (6), 2023-2030. doi:10.1111/cbdd.13604

Kang, L. (2020). Function Characteristics of Xuebijing in the Treatment of COVID-19. Pharmacology. Clin. Chin. Mater. Med. 36, 11-15. doi:10.13412/ j.cnki.zyyl.20200420.001

Kong, Y., Lin, L. L., Chen, Y., Lai, S., Wu, H. W., and Chen, J. S. (2020a). Mechanism of XueBijing Injection on Treatment of Coronavirus Disease 2019 Based on Network Pharmacology. Mater-world. Sci. Tech., 1-9. doi:10.11842/ wst.20200220003

Kong, Y., Wu, H. W., Chen, Y., Lai, S., Yang, Z. M., and Chen, J. S. (2020b). Mechanism of Tanreqing Injection on Treatment of Coronavirus Disease 2019 Based on Network Pharmacology and Molecular Docking. Chin. Tradit. Herb. Drugs 51, 1785-1794. doi:10.7501/j.issn.0253-2670.2020.07.012

Kuba, K., Imai, Y., Rao, S., Gao, H., Guo, F., Guan, B., et al. (2005). A Crucial Role of Angiotensin Converting Enzyme 2 (ACE2) in SARS Coronavirus-Induced Lung Injury. Nat. Med. 11 (8), 875-879. doi:10.1038/nm1267

Kumar, R., Afsar, M., Khandelwal, N., Chander, Y., Riyesh, T., Dedar, R. K., et al. (2021). Emetine Suppresses SARS-CoV-2 Replication by Inhibiting Interaction of Viral mRNA with eIF4E. Antiviral Res. 189, 105056. doi:10.1016/ j.antiviral.2021.105056

Leng, Z., Zhu, R., Hou, W., Feng, Y., Yang, Y., Han, Q., et al. (2020). Transplantation of ACE2- Mesenchymal Stem Cells Improves the Outcome of Patients with COVID19 Pneumonia. Aging Dis. 11 (2), 216-228. doi:10.14336/AD.2020.0228

Li, Y. C., Shen, J. D., Li, J., Wang, R., Jiao, S., and Yi, L. T. (2013). Chronic Treatment with Baicalin Prevents the Chronic Mild Stress-Induced DepressiveLike Behavior: Involving the Inhibition of Cyclooxygenase-2 in Rat Brain. Prog. Neuropsychopharmacol Biol. Psychiatry 40, 138-143. doi:10.1016/ j.pnpbp.2012.09.007

Li, P., Wang, T., Fu, S. F., Chang, N. W., and Wang, X. Y. (2016). Clinical Comparison of Shenfu Injection and Shenmai Injection Based on Meta Analysis. Chin. Tradit. Herb. Drugs 47 (16), 2949-2959. doi:10.7501/j.issn.0253-2670.2016.16.030

Li, Q., Yin, J., Ran, Q. S., Yang, Q., Liu, L., Zhao, Z., et al. (2019). [Efficacy and Mechanism of Lianhua Qingwen Capsules(LHQW) on Chemotaxis of Macrophages in Acute Lung Injury (ALI) Animal Model]. Zhongguo Zhong Yao Za Zhi 44 (11), 2317-2323. doi:10.19540/j.cnki.cjcmm.20190210.001

Li, Q., Guan, X., Wu, P., Wang, X., Zhou, L., Tong, Y., et al. (2020a). Early Transmission Dynamics in Wuhan, China, of Novel Coronavirus-Infected Pneumonia. N. Engl. J. Med. 382 (13), 1199-1207. doi:10.1056/NEJMoa2001316

Li, R. F., Hou, Y. L., Huang, J. C., Pan, W. Q., Ma, Q. H., Shi, Y. X., et al. (2020b). Lianhuaqingwen Exerts Anti-viral and Anti-inflammatory Activity against 
Novel Coronavirus (SARS-CoV-2). Pharmacol. Res. 156, 104761. doi:10.1016/ j.phrs.2020.104761

Li, W. N., Ba, Y. M., Wang, L. Q., Tao, R., Zuo, X. H., Shi, Q., et al. (2020c). The Role of Syndrome Differentiation in Traditional Chinese-western Medicine Combined Diagnosis and Treatment of 105 Cases of COVID-19. J. Jinan. Univ. 41 (5), 383-390. doi:10.11778/j.jdxb.2020.05.001

Li, Y. H., Yuan, Y., Wang, Y. W., and Zhao, M. (2021). The Role of PI3K/AKT-Mediated Apoptosis Signaling Pathway in Paraquat Poisoning-Induced Cardiac Injury. Chin. J. Diffic. Compl. Cas. 20 (3), 278-282. doi:10.3969/j.issn.1671-6450.2021.03.014

Li, Y. M., Tong, J. S., Li, Z. Y., Wang, J. Z., and Qin, X. L. (2020d). Mechanism of ShenFu Decoction in the Treatment of Critically Ill Patients with Corona Virus Disease 2019(COVID-19) Based on Network Pharmacology. J. Hainan. Med. Univ. 1-7. doi:10.13210/j.cnki.jhmu.20200525.004

Li, Z., Cao, H., Cheng, Y., Zhang, X., Zeng, W., Sun, Y., et al. (2020e). Inhibition of Porcine Epidemic Diarrhea Virus Replication and Viral 3C-like Protease by Quercetin. Ijms 21, 8095. doi:10.3390/ijms21218095

Liang, Y. F., and Zhou, Y. W. (2011). Studies on Antipyretic and Antiinflammatory Effects of Shuanghuanglian Oral Liquid. Chin. J. Clin. Ration. Drug Use. 4 (27), 51-52. doi:10.3969/j.issn.1674-3296.2011.27.041

Liao, P.-C., Lai, M.-H., Hsu, K.-P., Kuo, Y.-H., Chen, J., Tsai, M.-C., et al. (2018). Identification of $\beta$-Sitosterol as In Vitro Anti-inflammatory Constituent in Moringa Oleifera. J. Agric. Food Chem. 66 (41), 10748-10759. doi:10.1021/ acs.jafc. 8 b0 04555

Lin, J. R., Zheng, W. W., Zeng, G. X., and Lin, Q. C. (2020). Study on the Network Pharmacology of Jinhua Qinggan Granules in the Treatment of COVID-19. J. Chin. Med. Mater. 08, 2074-2080. doi:10.13863/j.issn1001-4454.2020.09

Ling, X. Y., Tao, J. L., Sun, X., and Yuan, B. (2020). Exploring Material Basis and Mechanism of Lianhua Qingwen Prescription against Coronavirus Based on Network Pharmacology. Chin. Tradit. Herb. Drugs 51, 1723-1730. doi:10.7501/ j.issn.0253-2670.2020.07.006

Ling, Y., Li, Z. Q., Yuan, K. H., Xiu, X. Q., Chen, J., He, G. W. W., et al. (2004). Small Molecules Blocking the Entry of Severe Acute Respiratory Syndrome coronavirus into Host Cells. J. Virol. 78 (20), 11334-11339. doi:10.1128/ JVI.78.20.11334-11339.200410.1128/jvi.78.20.11429-11433.2004

Liu, H. F., Zhao, Y. Y., Wei, F., and Chen, C. S. (2009). Protective Effect of Shengmai Injection to Acute Lung Injury Induced by Paraquat in Rats. Chin. J. Mod. Med. 19 (24), 3688-3691.

Liu, H., Ye, F., Sun, Q., Liang, H., Li, C., Li, S., et al. (2021). Scutellaria Baicalensis Extract and Baicalein Inhibit Replication of SARS-CoV-2 and its 3C-like Protease In Vitro. J. Enzyme Inhib. Med. Chem. 36 (1), 497-503. doi:10.1080/14756366.2021.1873977

Liu, J., Ma, L., Lu, J., Tian, X. M., Xu, J., Shen, X. P., et al. (2016). Study on Mechanism for Antipyretic Effects of Phufeng Giedu Capsule. Chin. Tradit. Herb. Drugs 47 (12), 2040-2043. doi:10.7501/j.issn.0253-2670.2016.12.004

Liu, K. F., Fu, M., Zhang, Z. P., and Zhang, Z. (2020a). Clinical Efficacy of Xuebijing in 2019 Patients with COVID-19. Nat. Confer. Sci. Res. Ttheory. Acad. Res. 395-398. doi:10.26914/c.cnkihy.2020.007030

Liu, Y. R., Tang, Z. S., Song, Z. X., Zhou, R., Xu, H. B., Yu, J. G., et al. (2020b). Feasibility Analysis of Siji Kangbingdu Mixture for Treatment of Children COVID-19. Mod. Chin. Med. 22 (4), 492-503. doi:10.13313/j.issn.16734890.20200311010

Loganathan, S., Kuppusamy, M., Wankhar, W., Gurugubelli, K. R., Mahadevappa, V. H., Lepcha, L., et al. (2021). Angiotensin-converting Enzyme 2 (ACE2): COVID 19 Gate Way to Multiple Organ Failure Syndromes. Respir. Physiol. Neurobiol. 283, 103548. doi:10.1016/j.resp.2020.103548

Loschwitz, J., Jäckering, A., Keutmann, M., Olagunju, M., Eberle, R. J., Coronado, M. A., et al. (2021). Novel Inhibitors of the Main Protease Enzyme of SARSCoV-2 Identified via Molecular Dynamics Simulation-Guided In Vitro Assay. Bioorg. Chem. 111, 104862. doi:10.1016/j.bioorg.2021.104862

Lu, R. B., Wang, W. J., and Li, X. (2020). Clinical Observation on Lianhua Qingwen Granules Combined with Western Medicine Conventional Therapy in the Treatment of 63 Suspected Cases of Coronavirus Disease 2019. J. Tradit. Chin. Med. 61 (8), 655-659. doi:10.13288/j.11-2166/r.2020.08.003

Ma, L.-L., Liu, H.-M., Luo, C.-H., He, Y.-N., Wang, F., Huang, H.-Z., et al. (2021). Fever and Antipyretic Supported by Traditional Chinese Medicine: A Multi-Pathway Regulation. Front. Pharmacol. 12, 583279. doi:10.3389/fphar.2021.583279

Ma, Z. G., Yu, J., Li, Y., and Cao, H. R. (2016). The Effects of Invigorating Lung and Activating Blood Circilation Capsule on Inflammatory Factors and
Immunologic Function of Patiments with Bronchial Asthma at Remission Stage. Lab. Med. Clin. 13, 2746-2748. doi:10.3969/j.issn.1672-9455.2016.19.019

Mao, Y., Sun, Y. X., Xue, P., Li, L. L., and Zhu, S. J. (2020). Discussion on the Mechanism of Jinhua Qinggan Granules in the Treatment of Novel Coronavirus Pneumonia. J. Chin. Med. Mater. 11, 2843-2849. doi:10.13863/ j.issn1001-4454.2020.11.046

Martin-Sancho, L., Lewinski, M. K., Pache, L., Stoneham, C. A., Yin, X., Becker, M. E., et al. (2021). Functional Landscape of SARS-CoV-2 Cellular Restriction. Mol. Cel. doi:10.1016/j.molcel.2021.04.008

National Health Commission of the People's Republic of China and National Administration of Traditional Chinese Medicine (2020). Guideline on Diagnosis and Treatment of COVID-19 (Trial 8th Edition). Chin. Med. 15 (10), 1494-1499. doi:10.3760/j.issn.1673-4777.2020.10.002

Noack, M., and Miossec, P. (2017). Selected Cytokine Pathways in Rheumatoid Arthritis. Semin. Immunopathol. 39 (4), 365-383. doi:10.1007/s00281-0170619-z

Nomura, T., Fukushi, M., Oda, K., Higashiura, A., Irie, T., and Sakaguchi, T. (2019). In Vitro Effects of Traditional Kampo Drugs and Their Constituent Crude Drugs on Influenza Virus Replication: Suppression of Viral Protein Synthesis by Glycyrrhizae Radix. Evid Based Complement Alternat Med. 2019, 3230906. doi:10.1155/2019/3230906

Paules, C. I., Marston, H. D., and Fauci, A. S. (2020). Coronavirus Infections-More Than Just the Common Cold. Jama 323 (8), 707-708. doi:10.1001/ jama.2020.0757

Pawlos, A., Niedzielski, M., Gorzelak-Pabiś, P., Broncel, M., and Woźniak, E. (2021). COVID-19: Direct and Indirect Mechanisms of Statins. Ijms 22 (8), 4177. doi:10.3390/ijms22084177

Petrera, E., Níttolo, A. G., and Alché, L. E. (2014). Antiviral Action of Synthetic Stigmasterol Derivatives on Herpes Simplex Virus Replication in Nervous CellsIn Vitro. Biomed. Res. Int. 2014, 1-9. doi:10.1155/2014/947560

Polidoro, R. B., Hagan, R. S., de Santis Santiago, R., and Schmidt, N. W. (2020), Overview: Systemic Inflammatory Response Derived from Lung Injury Caused by SARS-CoV-2 Infection Explains Severe Outcomes in COVID-19. Front. Immunol. 11, 1626. doi:10.3389/fimmu.2020.01626

Pu, D. Q., Liu, Z., Liang, J. W., Zhou, C., and Hao, Q. Z. (2020). Study of Reduning Injection Inhibiting Inflammatory Storm Based on Network Pharmacology and Molecular Docking in the Treatment of COVID-19. Pharm. Clin. Chin. Mater. Med., 1-17. doi:10.13412/j.cnki.zyyl.20200603.001

Qiao, Y. (2020). Effects of Qingxuan Zhike Granules on Anti Inflammation and Immunoregulation in Cough Rats. Chin. Tradit. Pat. Med. 42, 511-513. doi:10.3969/j.issn.1001-1528.2020.02.048

Qin, Y. L., Song, Y. Y., Zhou, L., Cui, Y., and Zhang, A. (2020). Clinical Efficacy of Reduning Injection Combined with Methylprednisolone in the Treatment of Severe Coronavirus Disease 2019. Chin. Pharm. 29, 19-22. doi:10.3969/ j.issn.1006-4931.2020.09.005

Qu, X. K., Hao, S. L., Ma, J. H., Wei, G. Y., Song, K. Y., Tang, C., et al. (2020). Observation on Clinical Effect of Shufeng Jiedu Capsule Combined with Arbidol Hydrochloride Capsule in Treatment of COVID-19. Chin. Tradit. Herb. Drugs 51 (5), 1167-1170. doi:10.7501/j.issn.0253-2670.2020.05.011

Ren, J.-1., Zhang, A.-H., and Wang, X.-J. (2020a). Traditional Chinese Medicine for COVID-19 Treatment. Pharmacol. Res. 155, 104743. doi:10.1016/ j.phrs.2020.104743

Ren, Q., Guo, F., Tao, S., Huang, R., Ma, L., and Fu, P. (2020b). Flavonoid Fisetin Alleviates Kidney Inflammation and Apoptosis via Inhibiting Src-Mediated NF-Kb P65 and MAPK Signaling Pathways in Septic AKI Mice. Biomed. Pharmacother. 122, 109772. doi:10.1016/j.biopha.2019.109772

Ren, Y., Yao, M. C., Huo, X. Q., Gu, Y., Zhu, W. X., Qiao, Y. J., et al. (2020c). [Study on Treatment of "cytokine Storm" by Anti-2019-nCoV Prescriptions Based on Arachidonic Acid Metabolic Pathway]. Zhongguo Zhong Yao Za Zhi 45 (6), 1225-1231. doi:10.19540/j.cnki.cjcmm.20200224.405

Ruan, B., Hu, H. F., and Yu, L. L. (2010). Effect of Qingxuan Zhike Granules on Acute Bronchitis Model Rats. Capital Med. 17, 43-44. doi:10.3969/j.issn.10058257.2010.06.033

Schwarz, S., Sauter, D., Wang, K., Zhang, R., Sun, B., Karioti, A., et al. (2014). Kaempferol Derivatives as Antiviral Drugs against the 3a Channel Protein of Coronavirus. Planta Med. 80, 177-182. doi:10.1055/s-0033-1360277

Shen, F., Fu, Z. Y., Wu, Y. R., Li, L., Zhao, Y. D., Xia, Y., et al. (2020). The Potential Molecular Mechanism of Active Compounds Binding SARS-CoV-2 Specific 
Target Proteins in Jinhua Qinggan Granules Treat COVID-19 Based on Network Pharmacology and High-Throughput Molecular Docking Fellowship. Modernization. Tradit. Chin. Med. Mater. Mater-world. Sci. Tech. 22, 622-631. doi:10.11842/wst.20200317010

Shi, C. Y., Lin, T., Wang, W. Q., and Fang, J. G. (2020a). Research Summary of Clearing Heat and Detoxification TCM Jinye Baidu Granules. Chin. Tradit. Herb. Drugs 51, 2055-2064. doi:10.7501/j.issn.0253-2670.2020.08.010

Shi, X., Wei, J., Liu, M. Y., Jin, X. H., Zhou, H. P., Zhu, W. L., et al. (2020b). Study on the Overall Regulation of Xuebijing Injection in Treating COVID-19. Shanghai J. Tradit. Chin. Med. 54, 46-52. doi:10.16305/j.1007-1334.2020.04.098

Shi, Y., Wang, X. P., Bai, J. Q., and Long, K. H. (2013). Study on Anti-bacterial, Anti-viral Effects of the Forsythia Suspensa. Mod. Chin. Med. 15 (11), 950-953.

Smith, E. C., Blanc, H., Vignuzzi, M., Denison, M. R., and Denison, M. R. (2013). Coronaviruses Lacking Exoribonuclease Activity Are Susceptible to Lethal Mutagenesis: Evidence for Proofreading and Potential Therapeutics. Plos. Pathog. 9 (8), e1003565. doi:10.1371/journal.ppat.1003565

Snijder, E. J., Bredenbeek, P. J., Dobbe, J. C., Thiel, V., Ziebuhr, J., Poon, L. L. M., et al. (2003). Unique and Conserved Features of Genome and Proteome of SARS-Coronavirus, an Early Split-Off from the Coronavirus Group 2 Lineage. J. Mol. Biol. 331 (5), 991-1004. doi:10.1016/s0022-2836(03)00865-9

Song, W., Gui, M., Wang, X., and Xiang, Y. (2018). Cryo-EM Structure of the SARS Coronavirus Spike Glycoprotein in Complex with its Host Cell Receptor ACE2. Plos Pathog. 14 (8), e1007236. doi:10.1371/journal.ppat.1007236

State Administration of Traditional Chinese Medicine (2020). Some Traditional Chinese Medicine Have Been Preliminarily Confirmed to Have Obvious Curative Effect, and the central Guidance Group Requires Timely Summary. Available at: http://www.satcm.gov.cn/xinxifabu/shizhengyaowen/2020-03-05/ 13617.html (Accessed April 25, 2020).[

Sun, M. J., Zhou, Y. H., and Sui, Y. (2005). Experimental Study of the Protecting Effect of Injection of Refined Qing Kai Ling on the Damnify of Rat Viscera Induced by Endotoxin. Chin. J. Basic Med. Tradit. Chin. Med. 11, 828-830. doi:10.3969/j.issn.1006-3250.2005.11.012

Sun, X. D., Yan, Y. H., Chu, W. W., Zhang, Y. T., and Ying, L. J. (2017). Effects of Xuebijing Injection on Tubular Apoptosis and Associated Protein Expression in Sepsis Induced Acute Kidney Injure Rats. Chin. J. Mod. Appl. Pharm. 34 (2), 210-214. doi:10.13748/j.cnki.issn1007-7693.2017.02.013

Tao, X. Q., Ke, Z. P., Zhang, X. Z., Deng, Y., Cao, Z. Y., Cao, L., et al. (2020). Investigate Mechanism of Jinzhen Oral Liquid for Prevention COVID-19 Based on Network Pharmacology and Molecular Docking Technology. Chin. Tradit. Herb. Drugs 51, 2326-2333. doi:10.7501/j.issn.0253-2670.2020.09.006

Wang, H., Jin, X. Y., Pang, B., Liu, C. X., Zheng, W. K., Yang, F. W., et al. (2020a). [Analysis on Clinical Study Protocols of Traditional Chinese Medicine for Coronavirus Disease 2019]. Zhongguo Zhong Yao Za Zhi 45 (6), 1232-1241. doi:10.19540/j.cnki.cjcmm.20200220.501

Wang, H., Wang, L. N., and Gu, W. (2020b). Effects of Shenfu Injection on Lung Injury and Biochemical Indexes of Mice with Immersion in Low Temperature Water. J. Navy. Med. 41 (3), 278-282. doi:10.3969/j.issn.1009-0754.2020.03.012

Wang, K. F., Xiao, W., and Wang, Z. Z. (2013). Antipyretic and Anti Inflammatory Effects of Reduning Injection and its Mechanism. Chin. J. Hosp. Pharm. 3, 1918-1922.

Wang, L. F., Li, H. T., Wang, Y., Liu, X. L., Chen, Q. G., Xu, J., et al. (2020d). Study on Mechanism of Shengmai Injection against Novel Coronavirus Pneumonia Based on Network Pharmacology and Molecular Docking Technology. Chin. Tradit. Herb. Drugs 51, 2977-2987. doi:10.7501/j.issn.0253-2670.2020.11.016

Wang, L., Wang, Z. H., Zhang, H. R., Yu, H. X., Yang, K., Fu, B. H., et al. (2020c). Study on the Network Pharmacology and Preliminary Evidence of Lianhua Qingwen in the Treatment of 2019-nCoV. J. Chin. Med. Mater. 43, 772-778. doi:10.13863/j.issn1001-4454.2020.03.049

Wang, M., Tang, Z. S., Liu, Y. R., Song, Z. X., Zhou, R., Xu, H. B., et al. (2020e). Mechanism Prediction and Anti-virus Compounds Selection of Reyanning Mixture in the Treatment of COVID-19 Based on Network Pharmacology and Molecular Docking Technologies. Mod. Chin. Med. 22, 484-491.

Wang, Y., Chen, L., Zheng, L., Ku, B. Q., Yu, R., and Zhang, X. F. (2021). Clinical Effects of Qingfei Paidu Decoction Combined with Conventional Treatment on Patients with Coronavirus Disease 2019. Chin. Tradit. Pat. Med. 43 (3), 656-659. doi:10.3969/j.issn.1001-1528.2021.03.017

Wang, Y. Y., Deng, W. P., and Li, X. Q. (2020f). Study on the Mechanism of Protective Effect of Shuanghuanglian Oral Liquid on C57BL/6J Nude Mice with
Acute Liver Failure Induced by Hepatitis Virus Type 3. Mod. J. Integ. Tradit. Chin. West. Med. 29 (9), 941-944. doi:10.3969/j.issn.1008-8849.2020.09.007

Wang, F. C., Shen, B. X., He, C. Y., Zhao, W. C., and Nie, S. L. (2020g). Clinical Efficacy of Lianhua Qingwen Granules and its Mechanism on COVID-19 Based on Network Pharmacology. Pharmacol. Clin. Chin. Mater. Clin. Med. 36 (2), 93-101. doi:10.13412/j.cnki.zyyl.20200318.001

Wang, Y. Z., Cui, X. L., Gao, Y. J., Guo, S. S., Wang, X. K., Huang, Y., et al. (2006). [Antivirus Effects of Extract from Gardenia]. Zhongguo Zhong Yao Za Zhi 31 (14), 1176-1178. doi:10.3321/j.issn:1001-5302.2006.14.013

World Health Organization (2020). Statement on the Second Meeting of the International Health Regulations (2005). Emergency Committee Regarding the Outbreak of Novel Coronavirus (2019-nCoV). Availableat: https://www.who. $\mathrm{int} /$ news-room/detail/30-01-2020-statement-on-the-second-meeting-of-theinternational-health-regulations-(2005)-emergency-committee-regarding-theoutbreak-of-novel-coronavirus-(2019-ncov (Accessed April 28, 2020).

World Health Organization (2020). Virtual Press Conference on COVID-19. Available at: https://www.who.int/docs/default- source/coronaviruse/ transcripts/who-audio-emergencies-coronavirus-press-conference-full-andfinal-11mar2020.pdf?sfvrsn=cb432bb3_2 (Accessed March 27, 2020).

Wrapp, D., Wang, N., Corbett, K. S., Goldsmith, J. A., Hsieh, C.-L., Abiona, O., et al. (2020). Cryo-EM Structure of the 2019-nCoV Spike in the Prefusion Conformation. Science 367 (2), 1260-1263. doi:10.1101/2020.02.11.944462

Wu, J. X., Hou, L. L., Liu, C. L., Zhang, L., and Yan, Y. X. (2018). Study on the Pharmacological Action of Antiviral Granule. Chin. J. Veter. Med. 54 (6), 118-120.

Xiao, W., Xu, L. L., Huo, C. C., Zhang, X. F., Sun, L., Wang, Z. Z., et al. (2008). Experimental Study on the Effect of Jinzhen Oral Liquid on SARS Virus. J. Nanjing Univ. Tradit. Chin. Med. 24, 343-344. doi:10.3969/j.issn.10005005.2008.05.019

Xiao, W., Zheng, L. S., Shang, Q., Liu, T., Sun, L., and Wang, Z. Z. (2009). Experimental Study on the Effect of Jinzhen Oral Liquid on Influenza A (H1N1) Virus. Chin. Tradit. Herb. Drugs 40, 1443-1445. doi:10.3321/j.issn:02532670.2009.09.033

Xie, L. H., Wang, J. Q., Lin, X. Y., Qin, T., and Hu, G. H. (2020). Network Pharmacological Analysis and Mechanism Prediction of Xingnaojing Injection in Treatment of Neurological Damage Caused by SARS-CoV-2 Chinese. Chin. Tradit. Herb. Drugs 51, 3211-3222. doi:10.7501/j.issn.0253-2670.2020.12.013

Xin, W. Y. (2013). Progress in Pharmacological Study and the Underlying Mechanism of Baicalein and Baicalin. Chin. J. New Drugs 6, 647-653.

Xu, H. B., Li, P. X., Ji, X. J., Mo, J. H., Huang, Y. F., Fang, C. S., et al. (2020a). Mechanism of Shufeng Jiedu Capsule in the Treatment of Novel Coronavirus Pneumonia Based on Network Pharmacology and Molecular Docking. J. Chin. Med. Mater. 1-1. doi:10.13863/j.issn1001-4454.2020.08.049

Xu, J. H., Xue, Y., Zhang, Y., and Lu, G. (2020c). Study on Mechanism of Shufeng Jiedu Capsule in Treating COVID-19 Based on Network Pharmacology. Chin. Tradit. Herb. Drugs 51, 2015-2023. doi:10.7501/j.issn.0253-2670.2020.08.005

$\mathrm{Xu}$, J., and Zhang, Y. (2020b). Traditional Chinese Medicine Treatment of COVID19. Complement. Therapies Clin. Pract. 39, 101165. doi:10.1016/ j.ctcp. 2020.101165

Xu, Z., Shi, L., Wang, Y. J., Zhang, J. Y., Huang, L., Zhang, C., et al. (2020d). A New Coronavirus Associated with Human Respiratory Disease in China. Nature 579 (7798), 265-269. doi:10.1038/s41586-020-2008-3

Xu, Z., Shi, L., Wang, Y., Zhang, J., Huang, L., Zhang, C., et al. (2020e). Pathological Findings of COVID-19 Associated with Acute Respiratory Distress Syndrome. Lancet Respir. Med. 8 (4), 420-422. doi:10.1016/S2213-2600(20)30076-X

Xu, Z. Y., Li, X. B., Zhang, H. Y., Zhang, S. Y., Yuan, Y. M., Wang, Q., et al. (2019). Study on Antipyretic Effect and its Mechanisms of APN on Lipopolysaccharide-Induced Febrile Rabbits. Tradit. Chin. Drug Res. Clin. Pharmacol. 6, 692-694. doi:10.19378/j.issn.1003-9783.2019.06.009

Yang, H. B., Li, Y. X., Cao, L. M., Feng, X. P., Zhang, J. J., Jiang, S. Y., et al. (2020a). 11 Cases of New Coronavirus Pneumonia Treated with Modified Decoction of Chinese Herbs Plus Western Medicine. Guangxi. J. Tradit. Chin. Med. 43 (3), 1-4. doi:10.3969/j.issn.1003-0719.2020.03.001

Yang, J. (2020b). Pondering over COVID-19 Prevented and Treated by Traditional Chinese Medicine. J. Nanjing. Univ. Tradit. Chin. Med. 36, 149-151. doi:10.14148/j.issn.1672-0482.2020.0149

Yang, L. A., and Luo, J. H. (2017). Study on the Effect of Andrographolide on Children with Bronchiolitis Clinical Curative Effect and Cytokines. Jiangxi. 
J. Tradit. Chin. Med. 52 (3), 192-194. doi:10.3969/j.issn.10062238.2017.03.002

Yang, M. B., Dang, S. S., Huang, S., Li, Y. J., and Guo, Y. L. (2020c). Multi-center Clinical Observation of Reyanning Mixture in Treatment of COVID-19. Chin. J. Exp. Tradit. Med. Form., 3-18. doi:10.13422/j.cnki.syfjx.20201321

Yao, Y. X., He, Z. X., Liu, X. F., He, Y. Z., Lei, Y., Zhang, S. T., et al. (2020). Potential Material Basis of Kangbingdu Granules for Treatment of Coronavirus Disease 2019 (COVID-19) Based on Network Pharmacology and Molecular Docking Technology. Chin. Tradit. Herb. Drugs 51, 1386-1396. doi:10.7501/j.issn.02532670.2020.06.003

Yu, H. Y., Ren, X. H., Qi, X. X., Zuo, Q., and Liu, D. (2020a). Efficacy Study of Arbidol, Qingfei Paidu Decoction, Lianhua Qingwen Capsule, and Jinye Baidu Granules in the Treatment of Mild/Moderate COVID-19 in a Fangcang Shelter Hospital. Pharmacology. Clin. Mater. Med. 36 (6), 2-6. doi:10.13412/ j.cnki.zyyl.20201110.005

Yu, S. S. (2019). Research Progress on Chemical Constituents, Pharmacological Effects and Clinical Applications of Jinzhen Oral Liguid as Pediatric Chinese Patent Medicine for Treating Cough and Phlegm. World Sci. Tech-modern. Tradit. Chin. Med. 21 (12), 2759-2763. doi:10.11842/wst.20181008002

Yu, S., Zhu, Y., Xu, J., Yao, G., Zhang, P., Wang, M., et al. (2021b). Glycyrrhizic Acid Exerts Inhibitory Activity against the Spike Protein of SARS-CoV-2. Phytomedicine 85, 153364. doi:10.1016/j.phymed.2020.153364

Yu, Y. R., Lu, P. F., Wang, H. X., and Huang, B. K. (2020c). Prevention and Treatment of COVID-19 with Traditional Chinese Medicine. J. Pharm. Pract. 38 (3), 202-206. doi:10.12206/j.issn.1006-0111.202003098

Zarrilli, G., Angerilli, V., Businello, G., Sbaraglia, M., Traverso, G., Fortarezza, F., et al. (2021). The Immunopathological and Histological Landscape of COVID19-Mediated Lung Injury. Ijms 22 (2), 974. doi:10.3390/ijms22020974

Zeng, X. H., Ma, W. H., and Wang, J. (2020). Effect of Qingfei Paidu Decoction on Clinical Efficacy of COVID-19 Pneumonia with Phlegm Heat Blocking Lung. Med. J. West. Chin. 32 (12), 1799-1801. doi:10.3969/j.issn.16723511.2020.12.017

Zhan, Y., Ta, W., Tang, W., Hua, R., Wang, J., Wang, C., et al. (2021). Potential Antiviral Activity of Isorhamnetin against SARS-CoV -2 Spike Pseudotyped Virus In Vitro. Drug Dev. Res. doi:10.1002/ddr.21815

Zhang, C. Y., Li, Z. H., Zhang, S., Wang, W., and Jiang, X. Q. (2020a). Clinical Observation of Xuebijing in the Treatment of COVID-19. Chin. J. Hosp. Pharm. 40, 964-967. doi:10.13286/j.1001-5213.2020.0903

Zhang, D., Zhang, B., Lv, J.-T., Sa, R.-N., Zhang, X.-M., and Lin, Z.-J. (2020b). The Clinical Benefits of Chinese Patent Medicines against COVID-19 Based on Current Evidence. Pharmacol. Res. 157, 104882. doi:10.1016/j.phrs.2020.104882

Zhang, F., Huang, J., Liu, W., Wang, C.-R., Liu, Y.-F., Tu, D.-Z., et al. (2021). Inhibition of Drug-Metabolizing Enzymes by Qingfei Paidu Decoction: Implication of Herb-Drug Interactions in COVID-19 Pharmacotherapy. Food Chem. Toxicol. 149, 111998. doi:10.1016/j.fct.2021.111998

Zhang, H., Liu, Y., and Li, J. S. (2015). Effect on Qingxuan Zhike Particles in the Treatment of Children with Upper Respiratory Infection. Chin. Med. Herald. $30,132-135$.

Zhang, Y. R., Gan, Z. Q., Liu, Z. X., Luo, J., Tang, C., Liu, C., et al. (2020c). Exploring Mechanism of Qingkailing Injection in Treatment of Coronavirus Disease 2019 (COVID-19) Based on Network Pharmacology and Molecular Docking. Chin. Tradit. Herb. Drugs 51, 3201-3210. doi:10.7501/j.issn.02532670.2020.12.012
Zhang, Y., and Yang, X. L. (2008). The Difference between Shengmai Injection and Shenmai Injection. J. Pract. Tradit. Chin. Med. 24 (10), 683. doi:10.3969/ j.issn.1004-2814.2008.10.066

Zhao, J., Tian, S., Lu, D., Yang, J., Zeng, H., Zhang, F., et al. (2021). Systems Pharmacological Study Illustrates the Immune Regulation, Anti-infection, Anti-inflammation, and Multi-Organ protection Mechanism of Qing-FeiPai-Du Decoction in the Treatment of COVID-19. Phytomedicine 85, 153315. doi:10.1016/j.phymed.2020.153315

Zhao, S. C., Wei, R. L., Xie, Y. M., Wang, L. X., Wang, Q., and Yi, D. H. (2019). [Analysis of Qingkailing Injection in Treatment of Combined Medication Features of 2147 Cases of Upper Respiratory Tract Infection]. Zhongguo Zhong Yao Za Zhi 44 (23), 5207-5216. doi:10.19540/ j.cnki.cjcmm.20191115.501

Zheng, Y., Liu, Z. Q., Zhu, X. Q., and Wang, B. L. (2020a). To Investigation of the Mechanism of Xuebijing Injection in COVID-19 Treatment Based on Network Pharmacology and Molecular Docking. Chin. J. Comp. Med. 1-13. doi:10.3969/ j.issn.1671-7856.2020.07.008

Zheng, Z. W., Ye, W. Q., and Liu, C. F. (2020b). Study on Mechanism of Shuanghuanglian Oral Liquid in Treating Based on Network Pharmacology. J. Chin. Med. Mater. 1-9. doi:10.13863/j.issn1001-4454.2020.06.045

Zhou, P., Yang, X.-L., Wang, X.-G., Hu, B., Zhang, L., Zhang, W., et al. (2020a). A Pneumonia Outbreak Associated with a New Coronavirus of Probable Bat Origin. Nature 579 (7798), 270-273. doi:10.1038/s41586-020-2012-7

Zhou, X., Lu, Q., Kang, X., Tian, G., Ming, D., Yang, J., et al. (2021). Protective Role of a New Polysaccharide Extracted from Lonicera japonica Thunb in Mice with Ulcerative Colitis Induced by Dextran Sulphate Sodium. Biomed. Res. Int. 2021, 1-9. doi:10.1155/2021/8878633

Zhou, Y., Fu, B., Zheng, X., Wang, D., Zhao, C., Qi, Y., et al. (2020b). Pathogenic T-Cells and Inflammatory Monocytes Incite Inflammatory Storms in Severe COVID-19 Patients. Nati. Sci. Rev. 7, 998-1002. doi:10.1093/nsr/nwaa041

Zhou, Y. X., Mei, B., Gao, J. F., and Liu, Z. Y. (2020c). Research Progress of Bufei Huoxue Capsule in the Treatment of Chronic Respiratory Diseases. Clin. J. Tradit. Chin. Med. 32 (12), 2364-2367. doi:10.16448/j.cjtcm.2020.1246

Zong, S. B., Sun, L., Lu, Y. Z., Zhou, J., Wang, Z. Z., and Xiao, W. (2018). Effect of Jinzhen Oral Liquid on NF-Kb, MAPK Signaling Pathway in Mice with LPSInduced Acute Lung Injury. Chin. J. Exper. Tradit. Med. Form. 24 (9), 155-159. doi:10.13422/j.cnki.syfjx.20180733

Zong, Y., Yao, W. F., Shan, J. J., and Wang, S. C. (2020). Discussion on the Active Compounds of Qingxuan Zhike Granule in Treatment of Pediatric Coronavirus Disease 2019 Based on Network Pharmacology and Molecular Docking Method. World Chin. Med. 15, 477-483. doi:10.3969/j.issn.1673-7202.2020.04.001

Conflict of Interest: The authors declare that the research was conducted in the absence of any commercial or financial relationships that could be construed as a potential conflict of interest.

Copyright $\odot 2021$ Yang, Wang, Ma, Wang, Lin, Yan, Fan, Chen, Ge, Zhu, Wang, Zhang, Sun and Feng. This is an open-access article distributed under the terms of the Creative Commons Attribution License (CC BY). The use, distribution or reproduction in other forums is permitted, provided the original author(s) and the copyright owner(s) are credited and that the original publication in this journal is cited, in accordance with accepted academic practice. No use, distribution or reproduction is permitted which does not comply with these terms. 